\title{
ESTIMATE OF THE PRESSURE WHEN ITS GRADIENT IS THE DIVERGENCE OF A MEASURE. APPLICATIONS
}

\author{
MARC BRIANe ${ }^{1}$ And JuAn CASADO-DÍAz ${ }^{2}$
}

\begin{abstract}
In this paper, a $W^{-1, N^{\prime}}$ estimate of the pressure is derived when its gradient is the divergence of a matrix-valued measure on $\mathbb{R}^{N}$, or on a regular bounded open set of $\mathbb{R}^{N}$. The proof is based partially on the Strauss inequality [Strauss, Partial Differential Equations: Proc. Symp. Pure Math. 23 (1973) 207-214] in dimension two, and on a recent result of Bourgain and Brezis [J. Eur. Math. Soc. 9 (2007) 277-315] in higher dimension. The estimate is used to derive a representation result for divergence free distributions which read as the divergence of a measure, and to prove an existence result for the stationary Navier-Stokes equation when the viscosity tensor is only in $L^{1}$.
\end{abstract}

Mathematics Subject Classification. 35Q30, 35Q35, 35A08.

Received February 22, 2010. Revised April 28, 2010.

Published online October 28, 2010.

\section{INTRODUCTION}

The estimate of the pressure is crucial for the mathematical analysis of incompressible fluid flow. We refer to $[14,15,17,20,21]$, for a general study of the problem. In particular, applying the distributional de Rham's theorem [10] to the framework of Sobolev spaces, one has the classical result (see, e.g., [20] for the case $m=1$, $r=2$, and its extension in [1]):

Theorem 1.1. Let $\Omega$ be a bounded Lipschitz-continuous connected open subset of $\mathbb{R}^{N}, N \geq 2$, let $m$ be a nonnegative integer, and let $r \in(1, \infty)$, with conjugate exponent $r^{\prime}:=\frac{r}{r-1}$. Then, for any distribution $F \in$ $W^{-m, r}(\Omega)^{N}$ satisfying

$$
\langle F, \Phi\rangle=0, \quad \forall \Phi \in C_{c}^{\infty}(\Omega)^{N}, \text { with } \operatorname{div}(\Phi)=0,
$$

there exists a unique $p \in W^{-m+1, r}(\Omega) / \mathbb{R}$ such that $F=\nabla p$. Moreover, there exists a constant $C>0$ which only depends on $\Omega, m, r$, such that

$$
\|p\|_{W^{-m+1, r}(\Omega) / \mathbb{R}} \leq C\|\nabla p\|_{W^{-m, r}(\Omega)^{N}}
$$

For instance, the previous estimate is a straightforward consequence of the fact (see, e.g., [3]) that any function $\varphi \in W_{0}^{m-1, r^{\prime}}(\Omega)\left(L^{r^{\prime}}(\Omega)\right.$ if $\left.m=1\right)$ with zero $\Omega$-average, is the divergence of a vector-valued function $\Phi \in$

\footnotetext{
Keywords and phrases. Pressure, Navier-Stokes equation, div-curl, measure data, fundamental solution.

${ }^{1}$ Institut de Recherche Mathématique de Rennes, INSA de Rennes, France. mbriane@insa-rennes.fr

2 Dpto. de Ecuaciones Diferenciales y Análisis Numérico, Universidad de Sevilla, Spain. jcasadod@us.es
} 
$W_{0}^{m, r^{\prime}}(\Omega)^{N}$ satisfying

$$
\|\Phi\|_{W_{0}^{m, r^{\prime}}(\Omega)^{N}} \leq C\|\varphi\|_{W^{m-1, r^{\prime}(\Omega)}} .
$$

These estimates are strongly connected to the Calderón-Zygmund inequality (see Thm. 1.2) which only holds for $r \in(1, \infty)$. Then, the natural question is to know if estimate (1.2) remains valid for $r=1$. In this critical case, the (badly defined) space $W^{-1,1}(\Omega)^{N}$ has to be regarded as the set of the divergence of the matrix-valued Radon measures on $\Omega$.

This question is motivated by the study of the pressure for the Stokes or Navier-Stokes equation (see Sect. 3), respectively for the incompressible elasticity, in a multi-fluid with high-contrast viscosity $\mu$, respectively in a multi-phase material with high-contrast rigidity, which is assumed to be only in $L^{1}$. Under this assumption the stress tensor (i.e. the product of $\mu$ by the strain tensor) also belongs to $L^{1}$, which makes delicate the estimate of the pressure since its gradient is expressed as the divergence of the stress tensor. Note that in the framework of homogenization theory the heterogeneous media with high-contrast phases may have various degenerate macroscopic behaviors (see, e.g., $[2,8,9,13,16,18]$ and the references therein). For example, the homogenization of the Stokes problem [7] is partly based on a pressure estimate for a very specific bi-fluid arranged along very thin cylinders with high viscosity, which leads to an effective nonlocal Brinkman law.

In a more general context our purpose is to give an estimate of the pressure $p$ when $\nabla p$ is the divergence of a matrix-valued measure $\mu \in \mathcal{M}(\Omega)^{N \times N}$. To this end, taking the Curl operator to eliminate the pressure $p$ we are led to estimate the measure $\mu$ assuming that its divergence is curl free. We then obtain an estimate of the measure $\mu-\frac{1}{N} \operatorname{tr}(\mu) I_{N}$ in the space $W^{-1, N^{\prime}}(\Omega)^{N \times N}$. This provides an estimate of $\nabla\left(p-\frac{1}{N} \operatorname{tr}(\mu)\right)$ in $W^{-2, N^{\prime}}(\Omega)^{N}$. Therefore, using the classical estimate (1.2) with $r=N^{\prime}$, we get an estimate of the pressure $p-\frac{1}{N} \operatorname{tr}(\mu)$ in $W^{-1, N^{\prime}}(\Omega)$.

First, the estimate of the pressure is obtained in the whole space $\mathbb{R}^{N}$ (see Thm. 2.1). Then, interior estimates are derived in the case of a bounded open set of $\mathbb{R}^{N}$ (see Thm. 2.7). Finally, boundary estimates are proved in the case of a regular bounded open $\Omega$ of $\mathbb{R}^{N}$ (see Thm. 2.8). Contrary to the classical estimate (1.2) which holds if $\Omega$ is only Lipschitz-continuous, here we need a $C^{3}$-regularity of $\Omega$, due to the change of variables we use in the second-order operator Curl (Div) around the boundary $\partial \Omega$ (see Lem. 2.11 whose proof is thereby rather delicate).

The proof of the pressure estimate in the whole space is very sensitive to the dimension. In dimension two the key ingredient is the Strauss inequality [19] which permits to bound the $L^{N^{\prime}}$-norm of a vector-valued function by the $L^{1}$-norm of its symmetrized gradient. However, since the algebra of the kernel of Curl (Div) is much more complicated in dimension three, we use an alternative approach which avoids partially tedious algebraic computations. This approach is based on a result due to Bourgain and Brezis [4], which states that there exists a constant $C_{N}$ only depending on $N$, such that any divergence free vector-valued function $f \in L^{1}\left(\mathbb{R}^{N}\right)^{N}$ satisfies

$$
\|D(\Gamma * f)\|_{L^{N^{\prime}}\left(\mathbb{R}^{N}\right)^{N \times N}} \leq C_{N}\|f\|_{L^{1}\left(\mathbb{R}^{N}\right)^{N}}
$$

where $\Gamma$ is the fundamental solution of the Laplace operator in $\mathbb{R}^{N}$. Estimate (1.4) and various extensions $[6,22]$ have remarkable applications to linear elliptic pde's. In particular, they allow to obtain generalizations of the Strauss inequality (see [4], Thm. 25), which establishes a connection with our two-dimensional approach of the pressure estimate. In higher dimension we use another extension of estimate (1.4) (see inequality (2.19)).

Therefore, the previous analysis can be regarded as a new application of [4] for dimension greater than two, the two-dimensional case being treated by a more elementary approach. As a consequence of the pressure estimate we can also answer to the starting question. Indeed, we obtain an extension of the classical Theorem 1.1 to $r=1, m=1$. More precisely, we prove a representation result for any distribution $F \in \mathcal{D}^{\prime}(\Omega)^{N}$ which is the divergence of a measure $\mu \in \mathcal{M}(\Omega)^{N \times N}$, and satisfies (1.1) (see Thm. 2.2 if $\Omega=\mathbb{R}^{N}$, and Thm. 2.9 if $\Omega$ is a regular bounded open set of $\left.\mathbb{R}^{N}\right)$. Actually, the representative pressure $p$ such that $F=\nabla p$, splits into a distribution in $W^{-1, N^{\prime}}(\Omega)$ and a measure in $\mathcal{M}(\Omega)$, the norm of each one of the two components being bounded by the norm of $\mu$. 
Finally, we give an application of the $W^{-1, N^{\prime}}$ pressure estimate to fluid mechanics. We prove the existence of a solution of the Navier-Stokes equation in a $C^{3}$-regular bounded open set $\Omega$ of $\mathbb{R}^{N}$, when the symmetric tensor-valued viscosity only belongs to $L^{1}(\Omega)^{N^{2} \times N^{2}}$. Moreover, we get an estimate of the stress tensor in the space $W^{-1, N^{\prime}}(\Omega)^{N \times N}$ (see Thm. 3.1).

\section{Notations and recalls}

- $\left(e_{1}, \ldots, e_{N}\right)$ denotes the canonic basis of $\mathbb{R}^{N}, N \geq 2$.

- $B(0, r)$ denotes the ball in $\mathbb{R}^{N}$ centered at the origin and of radius $r$, and $|B(0, r)|$ its Lebesgue measure.

- $I_{N}$ denotes the unit matrix of $\mathbb{R}^{N \times N}$, and tr denotes the trace of a matrix in $\mathbb{R}^{N \times N}$.

- The first-order derivative with respect to the variable $x_{i}$ is denoted $\partial_{i}$, and the second-order derivative with respect to the variables $x_{i}, x_{j}$ is denoted $\partial_{i j}^{2}$.

- For any $u: \mathbb{R}^{N} \longrightarrow \mathbb{R}^{n}, u=\left(u_{1}, \ldots, u_{n}\right), D u:=\left(\partial_{j} u_{i}\right)_{1 \leq i \leq n, 1 \leq j \leq N}, E(u):=\frac{1}{2}\left(D u+D u^{T}\right), D^{2} u=$ $\left(\partial_{j k}^{2} u_{i}\right)_{1 \leq i \leq n, 1 \leq j, k \leq N}$, and for $n=N, \operatorname{Curl}(u):=D u^{T}-D u$.

- For any $\mu: \mathbb{R}^{N} \longrightarrow \mathbb{R}^{N \times N}, \operatorname{Div}(\mu):=\left(\sum_{j=1}^{N} \partial_{j} \mu_{i j}\right)_{1<i<N}$.

- For any integer $k \geq 1$ and any $r \in(1, \infty), \hat{W}^{-k, r}\left(\mathbb{R}^{N}\right)$ denotes the subspace of the dual space $W^{-k, r}\left(\mathbb{R}^{N}\right)=\left(W^{k, r^{\prime}}\left(\mathbb{R}^{N}\right)\right)^{\prime}$, consisting in sums of partial derivatives of order $k$ of functions in $L^{r}\left(\mathbb{R}^{N}\right)$, and endowed with the norm

$$
\|f\|_{\hat{W}^{-k, r}\left(\mathbb{R}^{N}\right)}:=\inf \left\{|\langle f, \varphi\rangle|: \varphi \in C_{c}^{\infty}\left(\mathbb{R}^{N}\right), \text { with }\left\|D^{k} \varphi\right\|_{L^{r^{\prime}}\left(\mathbb{R}^{N}\right)^{N^{k}}}=1\right\} .
$$

Note that for a bounded open set $\Omega$ of $\mathbb{R}^{N}$, the corresponding space $\hat{W}^{-k, r}(\Omega)$ and $W^{-k, r}(\Omega)$ agree as a consequence of the Poincaré inequality (see, e.g., [5], Chap. 9).

- $c$ denotes a positive constant which may vary from line to line.

Along the paper we will use convolutions with the fundamental solution of the Laplace equation $\Gamma$ defined by

$$
\Gamma(x):=\left\{\begin{array}{cl}
\frac{-1}{N(N-2)|B(0,1)||x|^{N-2}} & \text { if } N>2 \\
\frac{\log |x|}{2 \pi} & \text { if } N=2,
\end{array} \quad \text { for } x \in \mathbb{R}^{N} \backslash\{0\} .\right.
$$

First of all, recall the Calderón-Zygmund inequality (see, e.g., [11], Thm. 9.9):

Theorem 1.2 (Calderón-Zygmund inequality). Let $\Omega$ be a bounded open set of $\mathbb{R}^{N}$, and let $r \in(1, \infty)$. Then, for any $f \in L^{r}\left(\mathbb{R}^{N}\right)$, with compact support in $\Omega$, there exist two constants $C_{\Omega}>0$ and $C>0$, such that $\Gamma * f$ belongs to $W^{2, r}(\Omega)$, with

$$
\|\Gamma * f\|_{W^{2, r}(\Omega)} \leq C_{\Omega}\|f\|_{L^{r}\left(\mathbb{R}^{N}\right)} \quad \text { and } \quad\left\|D^{2} \Gamma * f\right\|_{L^{r}\left(\mathbb{R}^{N}\right)^{N \times N}} \leq C\|f\|_{L^{r}\left(\mathbb{R}^{N}\right)},
$$

and $C$ does not depend on $\Omega$.

From the second inequality of (1.6) (the so-called Calderón-Zygmund inequality), we deduce that for any $f \in L^{r}\left(\mathbb{R}^{N}\right)$, with $r \in(1, \infty)$, and any sequence $f_{n}$ with compact support and strongly converging to $f$ in $L^{r}\left(\mathbb{R}^{N}\right)$, the convolution $D^{2} \Gamma * f$ is well defined by the limit

$$
D^{2} \Gamma * f:=\lim _{n \rightarrow \infty} D^{2} \Gamma * f_{n} \quad \text { strongly in } L^{r}\left(\mathbb{R}^{N}\right)^{N \times N},
$$


which is independent of the sequence $f_{n}$. In particular we have $\Delta \Gamma * f=f$. The definition (1.7) combined again with the Calderón-Zygmund inequality provides the following Calderón-Zygmund estimates in the spaces $\hat{W}^{-k, r}\left(\mathbb{R}^{N}\right)$ :

Corollary 1.3 (Calderón-Zygmund estimates). Let $k$ be a nonnegative integer and let $r \in(1, \infty)$. Then, there exists a constant $C>0$ such that the following estimates hold

$$
\forall f \in \hat{W}^{-k, r}\left(\mathbb{R}^{N}\right), \quad\left\{\begin{aligned}
\left\|D^{2} \Gamma * f\right\|_{\hat{W}^{-k, r}\left(\mathbb{R}^{N}\right)^{N \times N}} \leq C\|f\|_{\hat{W}^{-k, r}\left(\mathbb{R}^{N}\right)} & \text { if } k \geq 0, \\
\|D \Gamma * f\|_{\hat{W}^{-k+1, r}\left(\mathbb{R}^{N}\right)^{N}} \leq C\|f\|_{\hat{W}^{-k, r}\left(\mathbb{R}^{N}\right)} & \text { if } k \geq 1, \\
\|\Gamma * f\|_{\hat{W}^{-k+2, r}\left(\mathbb{R}^{N}\right)} & \leq C\|f\|_{\hat{W}^{-k, r}\left(\mathbb{R}^{N}\right)} \quad \text { if } k \geq 2 .
\end{aligned}\right.
$$

Actually, estimates (1.8) will be used for distributions $f$ with compact support (see the proofs of Lem. 2.5 and Thm. 2.7) so that the equalities $D \Gamma * f=\Gamma * D f=D(\Gamma * f)$ hold.

\section{THE MAIN RESUlts}

\subsection{The case of the whole space}

In this section we prove that any matrix-valued measure on $\mathbb{R}^{N}$, with zero trace, the divergence of which is curl free belongs actually to $\hat{W}^{-1, N}\left(\mathbb{R}^{N}\right)^{N \times N}$. This result has an interesting consequence on the representation of vector-valued distributions which vanish on the set of divergence free functions of $C_{c}^{\infty}\left(\mathbb{R}^{N}\right)^{N}$. The main result is the following:

Theorem 2.1. Let $\mu$ be a matrix-valued measure in $\mathcal{M}\left(\mathbb{R}^{N}\right)^{N \times N}$ which satisfies

$$
\operatorname{Curl}(\operatorname{Div}(\mu))=0 \quad \text { in } \mathcal{D}^{\prime}\left(\mathbb{R}^{N}\right)^{N \times N} .
$$

Then, there exists a constant $C_{N}>0$ only depending on $N$, such that $\mu-\frac{1}{N} \operatorname{tr}(\mu) I_{N}$ belongs to $\hat{W}^{-1, N}\left(\mathbb{R}^{N}\right)$ and

$$
\left\|\mu-\frac{1}{N} \operatorname{tr}(\mu) I_{N}\right\|_{\hat{W}^{-1, N^{\prime}}\left(\mathbb{R}^{N}\right)^{N \times N}} \leq C_{N}\|\mu\|_{\mathcal{M}\left(\mathbb{R}^{N}\right)^{N \times N}} .
$$

As a consequence of Theorem 2.1 we obtain the following result which can be used in fluid mechanics to prove the existence of the pressure when the viscosity term is known to be the divergence of a measure.

Theorem 2.2. Consider a distribution $F \in \mathcal{D}^{\prime}\left(\mathbb{R}^{N}\right)^{N}$ such that

$$
\langle F, \varphi\rangle=0, \quad \forall \varphi \in C_{c}^{\infty}\left(\mathbb{R}^{N}\right)^{N}, \text { with } \operatorname{div}(\varphi)=0,
$$

and such that there exists $M>0$ satisfying

$$
\langle F, \varphi\rangle \leq M\|D \varphi\|_{L^{\infty}\left(\mathbb{R}^{N}\right)^{N \times N}}, \quad \forall \varphi \in C_{c}^{\infty}\left(\mathbb{R}^{N}\right)^{N} .
$$

Then, there exist a distribution $p \in \hat{W}^{-1, N^{\prime}}\left(\mathbb{R}^{N}\right)$, a measure $q \in \mathcal{M}\left(\mathbb{R}^{N}\right)$ and a constant $C>0$ such that

$$
\begin{gathered}
F=\nabla(p+q) \quad \text { in } \mathcal{D}^{\prime}\left(\mathbb{R}^{N}\right)^{N}, \\
\|p\|_{\hat{W}^{-1, N^{\prime}}\left(\mathbb{R}^{N}\right)}+\|q\|_{\mathcal{M}\left(\mathbb{R}^{N}\right)} \leq C M .
\end{gathered}
$$

Moreover, if F satisfies

$$
\langle F, \varphi\rangle \leq M \inf \left\{\left\|D \varphi-h I_{N}\right\|_{L^{\infty}\left(\mathbb{R}^{N}\right)}: h \in C_{0}^{0}\left(\mathbb{R}^{N}\right)\right\}, \quad \forall \varphi \in C_{c}^{\infty}\left(\mathbb{R}^{N}\right),
$$

then we can take $q=0$. 
Corollary 2.3. There exists a constant $C>0$ such that for any distribution $g \in \mathcal{D}^{\prime}\left(\mathbb{R}^{N}\right)$ satisfying $\nabla g=\operatorname{Div}(\mu)$ in $\mathcal{D}^{\prime}\left(\mathbb{R}^{N}\right)$, with $\mu \in \mathcal{M}\left(\mathbb{R}^{N}\right)^{N \times N}$, there exists a constant $R \in \mathbb{R}$ such that

$$
g-\frac{1}{N} \operatorname{tr}(\mu)-R \in \hat{W}^{-1, N^{\prime}}\left(\mathbb{R}^{N}\right), \text { with }\left\|g-\frac{1}{N} \operatorname{tr}(\mu)-R\right\|_{\hat{W}^{-1, N^{\prime}\left(\mathbb{R}^{N}\right)}} \leq C\|\mu\|_{\mathcal{M}\left(\mathbb{R}^{N}\right)^{N \times N}} .
$$

Proof of Theorem 2.1. Using a regularization argument we can assume that $\mu$ belongs to $C^{\infty}\left(\mathbb{R}^{N}\right)^{N \times N}$. We distinguish the case $N=2$ from the case $N>2$.

The case $N=2$. By (2.1) there exists $p \in C^{\infty}\left(\mathbb{R}^{2}\right)$ defined up to an additive constant, such that

$$
\operatorname{Div}\left(\mu-\frac{1}{2} \operatorname{tr}(\mu) I_{2}-p I_{2}\right)=0
$$

Therefore, there exists a current function $z=\left(z_{1}, z_{2}\right) \in C^{\infty}\left(\mathbb{R}^{2}\right)^{2}$ such that

$$
\mu-\frac{1}{2} \operatorname{tr}(\mu) I_{2}-p I_{2}=D z J, \quad \text { where } J:=\left(\begin{array}{cc}
0 & -1 \\
1 & 0
\end{array}\right)
$$

which can read as

or equivalently,

$$
\left\{\begin{aligned}
\mu_{11}-\frac{1}{2} \operatorname{tr}(\mu)-p & =\partial_{2} z_{1} \\
\mu_{12} & =-\partial_{1} z_{1} \\
\mu_{21} & =\partial_{2} z_{2} \\
\mu_{22}-\frac{1}{2} \operatorname{tr}(\mu)-p & =-\partial_{1} z_{2}
\end{aligned}\right.
$$

$$
E(z)=\left(\begin{array}{cc}
-\mu_{12} & \frac{1}{2}\left(\mu_{11}-\mu_{22}\right) \\
\frac{1}{2}\left(\mu_{11}-\mu_{22}\right) & \mu_{21}
\end{array}\right) \quad \text { and } \quad p=\frac{1}{2}\left(\partial_{1} z_{2}-\partial_{2} z_{1}\right) .
$$

The matrix-valued $E(z)$ belongs to $L^{1}\left(\mathbb{R}^{2}\right)^{2 \times 2}$ and its norm is bounded by that of $\mu$. Hence, by the extension of the Korn inequality due to Strauss [19] there exist a constant $c>0$ independent of $z$ and a $z$-dependent rigid motion $r(x)=\alpha J x+a$, with $\alpha \in \mathbb{R}$ and $a \in \mathbb{R}^{2}$, such that

$$
\|z-r\|_{L^{2}\left(\mathbb{R}^{2}\right)^{2}} \leq c\|E(z)\|_{L^{1}\left(\mathbb{R}^{2}\right)^{2 \times 2}} \leq C\|\mu\|_{L^{1}\left(\mathbb{R}^{2}\right)^{2 \times 2}} .
$$

On the other hand, since $\operatorname{Dr} J=-\alpha I_{2}$, we have by (2.10)

$$
p I_{2}+D z J=\frac{1}{2}\left(\partial_{1}(z-r)_{2}-\partial_{2}(z-r)_{1}\right) I_{2}+D(z-r) J .
$$

This combined with (2.9) implies that

$$
\mu-\frac{1}{2} \operatorname{tr}(\mu) I_{2}=\frac{1}{2}\left(\partial_{1}(z-r)_{2}-\partial_{2}(z-r)_{1}\right) I_{2}+D(z-r) J .
$$

Finally, (2.12) and (2.11) yield the desired estimate (2.2) for $N=N^{\prime}=2$.

The case $N>2$. Assumption (2.1) can read as

$$
\forall i, j \in\{1, \ldots, N\}, \quad \partial_{j}\left(\sum_{k=1}^{N} \partial_{k} \mu_{i k}\right)-\partial_{i}\left(\sum_{k=1}^{N} \partial_{k} \mu_{j k}\right)=0 \quad \text { in } \mathcal{D}^{\prime}\left(\mathbb{R}^{N}\right),
$$

or equivalently,

$$
\forall i, j \in\{1, \ldots, N\}, \quad \operatorname{div}\left[\left(\partial_{j} \mu_{i k}-\partial_{i} \mu_{j k}\right)_{1 \leq k \leq N}\right]=0 \quad \text { in } \mathcal{D}^{\prime}\left(\mathbb{R}^{N}\right) .
$$

At this point we need the following result which is a simple adaptation to second-order derivatives of an estimate due to Van Schaftingen [23] (Thm. 1.5). For the reader's convenience it is proved in Appendix A.1. 
Lemma 2.4. Let $g \in L^{1}\left(\mathbb{R}^{N}\right)^{N \times N}$ the first column of which is zero, i.e. $g_{i 1}=0$ for any $i=1, \ldots, N$, and define the distribution $f:=\operatorname{Div}(g)$. Assume that $f$ is divergence free. Then, there exists a constant $C>0$ only depending on $N$, such that

$$
\forall u_{1} \in W^{2, N}\left(\mathbb{R}^{N}\right), \quad\left|\int_{\mathbb{R}^{N}} f_{1} u_{1} \mathrm{~d} x\right| \leq C\|g\|_{L^{1}\left(\mathbb{R}^{N}\right)^{N \times N}}\left\|D^{2} u_{1}\right\|_{L^{N}\left(\mathbb{R}^{N}\right)^{N \times N}} .
$$

Let $i, j, k_{0}$ be three distinct elements of $\{1, \ldots, N\}$. Define $g=\left(g_{1}, \ldots, g_{N}\right) \in L^{1}\left(\mathbb{R}^{N}\right)^{N \times N}$ by $g_{k}:=$ $\mu_{i k} e_{j}-\mu_{j k} e_{i}$, and $f=\left(f_{1}, \ldots, f_{N}\right)$ by $f_{k}:=\operatorname{div}\left(g_{k}\right)=\partial_{j} \mu_{i k}-\partial_{i} \mu_{j k}$, for $k=1, \ldots, N$. We have $g_{k k_{0}}=0$ for any $k=1, \ldots, N$, and by $(2.13) \operatorname{div}(f)=0$. Hence by Lemma 2.4 (replacing 1 by $k_{0}$ ) $f_{k_{0}}$ belongs to $\hat{W}^{-2, N^{\prime}}\left(\mathbb{R}^{N}\right)$, and there exists a constant $c>0$ only depending on $N$, such that

$$
\left\|f_{k_{0}}\right\|_{\hat{W}^{-2, N^{\prime}\left(\mathbb{R}^{N}\right)}} \leq c\|\mu\|_{L^{1}\left(\mathbb{R}^{N}\right)^{N \times N}} .
$$

Since $f_{k_{0}}=\operatorname{div}\left(g_{k_{0}}\right)$ with $g_{k_{0}} \in L^{1}\left(\mathbb{R}^{N}\right)^{N \times N}$, we deduce from Theorem 4' of [4] that there exists a constant $c>0$ only depending on $N$, such that

$$
\left\|g_{k_{0}}\right\|_{\hat{W}^{-1, N^{\prime}\left(\mathbb{R}^{N}\right)^{N}}} \leq c\left(\left\|g_{k_{0}}\right\|_{L^{1}\left(\mathbb{R}^{N}\right)^{N}}+\left\|f_{k_{0}}\right\|_{\hat{W}^{-2, N^{\prime}\left(\mathbb{R}^{N}\right)}}\right) \leq c\|\mu\|_{L^{1}\left(\mathbb{R}^{N}\right)^{N \times N}} .
$$

Recall that $g_{k_{0}}=\mu_{i k_{0}} e_{j}-\mu_{j k_{0}} e_{i}$, the integers $i, j, k_{0}$ being distinct. Then, noting that (since $N \geq 3$ ) for any indices $i \neq k$ there exists an index $j$ which is different from $i$ and $k$, the previous estimate implies that there exists a constant $C>0$ only depending on $N$, such that

$$
\forall i \neq k \in\{1, \ldots, N\}, \quad\left\|\mu_{i k}\right\|_{\hat{W}^{-1, N^{\prime}\left(\mathbb{R}^{N}\right)^{N}}} \leq C\|\mu\|_{L^{1}\left(\mathbb{R}^{N}\right)^{N \times N}}
$$

Now, let us estimate $\mu_{i i}-\mu_{j j}$ for $i \neq j$. By (2.1) we have

$$
\begin{aligned}
0 & =\sum_{k=1}^{N} \partial_{k}\left(\partial_{j} \mu_{i k}-\partial_{i} \mu_{j k}\right) \\
& =\partial_{i}\left(\partial_{j} \mu_{i i}-\partial_{i} \mu_{j i}\right)+\partial_{j}\left(\partial_{j} \mu_{i j}-\partial_{i} \mu_{j j}\right)+\sum_{k \neq i, j}^{N} \partial_{k}\left(\partial_{j} \mu_{i k}-\partial_{i} \mu_{j k}\right) \\
& =\partial_{i j}^{2}\left(\mu_{i i}-\mu_{j j}\right)+\partial_{j j}^{2} \mu_{i j}-\partial_{i i}^{2} \mu_{j i}+\sum_{k \neq i, j}^{N}\left(\partial_{j k}^{2} \mu_{i k}-\partial_{i k}^{2} \mu_{j k}\right) .
\end{aligned}
$$

This combined with the estimate (2.15) satisfied by $\mu_{k l}$ for any $k \neq l$, implies that there exists a constant $c>0$ only depending on $N$, such that

$$
\left\|\partial_{i j}^{2}\left(\mu_{i i}-\mu_{j j}\right)\right\|_{\hat{W}^{-3, N^{\prime}}\left(\mathbb{R}^{N}\right)} \leq c\|\mu\|_{L^{1}\left(\mathbb{R}^{N}\right)^{N \times N}} .
$$

On the other hand, making the change of variables

$$
\mu^{\prime}\left(x^{\prime}\right):=\mu(x), \quad \text { with }\left\{\begin{array}{l}
x_{i}^{\prime}:=x_{i}+x_{j} \\
x_{j}^{\prime}:=x_{i}-x_{j} \\
x_{k}^{\prime}:=x_{k}
\end{array} \text { if } k \neq i, j,\right.
$$

and $\partial_{i}^{\prime}$ denoting the derivative with respect to the variable $x_{i}^{\prime}$, we obtain that

$$
\partial_{i j}^{2}\left(\mu_{i i}-\mu_{j j}\right)=\partial_{i i}^{\prime}\left(\mu_{i i}^{\prime}-\mu_{j j}^{\prime}\right)-\partial_{j j}^{\prime}\left(\mu_{i i}^{\prime}-\mu_{j j}^{\prime}\right)=\sum_{k=1}^{N} \partial_{k k}^{\prime} u_{k k}^{\prime}
$$


where

$$
\left\{\begin{array}{l}
u_{i}^{\prime}:=\mu_{i i}^{\prime}-\mu_{j j}^{\prime} \\
u_{j}^{\prime}:=\mu_{j j}^{\prime}-\mu_{i i}^{\prime} \\
u_{k}^{\prime}:=0
\end{array} \text { if } k \neq i, j .\right.
$$

By $(2.18),(2.17),(2.16)$ we have $u^{\prime}=\left(u_{1}^{\prime}, \ldots, u_{N}^{\prime}\right) \in L^{1}\left(\mathbb{R}^{N}\right)^{N}$ and $\sum_{k=1}^{N} \partial_{k k}^{\prime} u_{k}^{\prime} \in \hat{W}^{-3, N^{\prime}}\left(\mathbb{R}^{N}\right)$. Then, by virtue of the Corollary 24' of [4] there exists a constant $c>0$ only depending on $N$, such that

$$
\left\|u^{\prime}\right\|_{\hat{W}^{-1, N^{\prime}}\left(\mathbb{R}^{N}\right)^{N}} \leq c\left(\left\|u^{\prime}\right\|_{L^{1}\left(\mathbb{R}^{N}\right)^{N}}+\left\|\sum_{k=1}^{N} \partial_{k k}^{\prime} u_{k}^{\prime}\right\|_{\hat{W}^{-3, N^{\prime}}\left(\mathbb{R}^{N}\right)}\right),
$$

which combined with (2.16) and (2.17) gives

$$
\left\|u^{\prime}\right\|_{\hat{W}^{-1, N^{\prime}}\left(\mathbb{R}^{N}\right)^{N}} \leq c\|\mu\|_{L^{1}\left(\mathbb{R}^{N}\right)^{N \times N}} .
$$

Therefore, taking into account the definition (2.18) of $u^{\prime}$ the previous estimate implies that there exists a constant $C>0$ only depending on $N$, such that

$$
\forall i, j \in\{1, \ldots, N\}, \quad\left\|\mu_{i i}-\mu_{j j}\right\|_{\hat{W}^{-1, N^{\prime}}\left(\mathbb{R}^{N}\right)^{N}} \leq C\|\mu\|_{L^{1}\left(\mathbb{R}^{N}\right)^{N \times N}} .
$$

Finally, noting that

$$
\mu_{i i}-\frac{1}{N} \operatorname{tr}(\mu)=\frac{1}{N} \sum_{j=1}^{N}\left(\mu_{i i}-\mu_{j j}\right),
$$

estimates (2.15) and (2.20) yield the desired result (2.2)

Proof of Theorem 2.2. If $F=\left(F_{1}, \ldots, F_{N}\right)$ is a distribution which satisfies (2.4), then an easy application of the Hahn-Banach theorem shows that there exists $\mu \in \mathcal{M}\left(\mathbb{R}^{N}\right)^{N \times N}$ such that

$$
F=\operatorname{Div}(\mu) \text { in } \mathcal{D}^{\prime}\left(\mathbb{R}^{N}\right)^{N}, \quad \text { with }\|\mu\|_{\mathcal{M}\left(\mathbb{R}^{N}\right)^{N \times N}} \leq M
$$

If $F$ also satisfies (2.3), then for any $\varphi \in C^{\infty}\left(\mathbb{R}^{N}\right)$ and any $i, j \in\{1, \ldots, N\}$, we have

$$
\left\langle(\operatorname{Curl}(F))_{i j}, \varphi\right\rangle=\langle F, \Phi\rangle=0
$$

where $\Phi=\left(\Phi_{1}, \ldots, \Phi_{N}\right) \in C_{c}^{\infty}\left(\mathbb{R}^{N}\right)^{N}$ is the divergence free function defined by

$$
\Phi_{i}:=\partial_{j} \varphi, \quad \Phi_{j}:=-\partial_{i} \varphi, \quad \Phi_{k}:=0 \text { if } k \neq i, j .
$$

Thus, condition (2.3) implies that $\operatorname{Curl}(F)=0$. Moreover, by (2.21) we get that

$$
\operatorname{Curl}(\operatorname{Div}(\mu))=0 \quad \text { in } \mathcal{D}^{\prime}\left(\mathbb{R}^{N}\right)^{N \times N} .
$$

Therefore, applying Theorem 2.1 it follows that $\nu:=\mu-\frac{1}{N} \operatorname{tr}(\mu) I_{N}$ belongs to $\hat{W}^{-1, N^{\prime}}\left(\mathbb{R}^{N}\right)$ and satisfies

$$
\|\nu\|_{\hat{W}^{-1, N^{\prime}}\left(\mathbb{R}^{N}\right)} \leq c\|\mu\|_{\mathcal{M}\left(\mathbb{R}^{N}\right)^{N \times N}} .
$$

On the other hand, taking into account that

$$
\operatorname{Div}(\nu)=\operatorname{Div}(\mu)-\nabla\left(\frac{1}{N} \operatorname{tr}(\mu)\right)
$$


we get that, analogously to $\mu, \nu$ satisfies

$$
\operatorname{Curl}(\operatorname{Div}(\nu))=0 \quad \text { in } \mathcal{D}^{\prime}\left(\mathbb{R}^{N}\right)^{N \times N} .
$$

We need the following result which is proved at the end of the section:

Lemma 2.5. Let $k$ be a positive integer and let $r \in(1, \infty)$. Then, there exists a constant $C>0$ which only depends on $k, r$ and $N$, such that any distribution $F \in \hat{W}^{-k, r}\left(\mathbb{R}^{N}\right)^{N}$ can be decomposed as

$$
F=\nabla p+G \quad \text { in } \mathcal{D}^{\prime}\left(\mathbb{R}^{N}\right)^{N}
$$

where $p \in \hat{W}^{-k+1, r}\left(\mathbb{R}^{N}\right)$ and $G \in \hat{W}^{-k, r}\left(\mathbb{R}^{N}\right)^{N}$ satisfy

$$
\begin{gathered}
\operatorname{div}(G)=0 \quad \text { in } \mathcal{D}^{\prime}\left(\mathbb{R}^{N}\right), \\
\begin{cases}\|p\|_{\hat{W}^{-k+1, r}\left(\mathbb{R}^{N}\right)^{N}} & \leq C\|\operatorname{div}(F)\|_{\hat{W}^{-k-1, r}\left(\mathbb{R}^{N}\right)} \\
\|G\|_{\hat{W}^{-k, r}\left(\mathbb{R}^{N}\right)^{N}} & \leq C\|\operatorname{Curl}(F)\|_{\hat{W}^{-k-1, r}\left(\mathbb{R}^{N}\right)^{N \times N}} .\end{cases}
\end{gathered}
$$

Applying Lemma 2.5 to $F:=\operatorname{Div}(\nu) \in \hat{W}^{-2, N^{\prime}}\left(\mathbb{R}^{N}\right)$, by $(2.23)$, (2.22) there exists a distribution $p \in$ $\hat{W}^{-1, N^{\prime}}\left(\mathbb{R}^{N}\right)$ such that

$$
\nabla p=\operatorname{Div}(\nu) \text { in } \mathcal{D}^{\prime}\left(\mathbb{R}^{N}\right)^{N}, \quad \text { with } \quad\|p\|_{\hat{W}^{-1, N^{\prime}}\left(\mathbb{R}^{N}\right)} \leq c\|\nu\|_{\hat{W}^{-1, N^{\prime}\left(\mathbb{R}^{N}\right)}} \leq C\|\mu\|_{\mathcal{M}\left(\mathbb{R}^{N}\right)^{N \times N}} .
$$

Then, using that

$$
F=\operatorname{Div}(\mu)=\operatorname{Div}(\nu)+\nabla\left(\frac{1}{N} \operatorname{tr}(\mu)\right)=\nabla\left(p+\frac{1}{N} \operatorname{tr}(\mu)\right) \quad \text { in } \mathcal{D}^{\prime}\left(\mathbb{R}^{N}\right)^{N},
$$

and by $(2.21)$

we obtain (2.6) just taking $q:=\frac{1}{N} \operatorname{tr}(\mu)$.

$$
\left\|\frac{1}{N} \operatorname{tr}(\mu)\right\|_{\mathcal{M}\left(\mathbb{R}^{N}\right)} \leq\|\mu\|_{\mathcal{M}\left(\mathbb{R}^{N}\right)^{N \times N}} \leq M,
$$

In order to conclude the proof of Theorem 2.2, we remark that condition (2.7) is equivalent to say that the functional

defined by

$$
\tilde{F}:\left\{D \varphi: \varphi \in C_{c}^{\infty}\left(\mathbb{R}^{N}\right)^{N}\right\} /\left\{h I_{N}: h \in C_{0}^{0}\left(\mathbb{R}^{N}\right)\right\} \longrightarrow \mathbb{R}
$$

$$
\tilde{F}[D \varphi]:=\langle F, \varphi\rangle, \quad \forall \varphi \in C_{c}^{\infty}\left(\mathbb{R}^{N}\right),
$$

(where $[D \varphi]$ denotes the class corresponding to $D \varphi$ ) is well defined and it is continuous with $\left\{D \varphi: \varphi \in C_{c}^{\infty}\left(\mathbb{R}^{N}\right)^{N}\right\}$ endowed with the uniform topology. By the Hahn-Banach theorem $\tilde{F}$ can be extended to a continuous functional defined in

$$
C_{0}^{0}\left(\mathbb{R}^{N}\right)^{N \times N} /\left\{h I_{N}: h \in C_{0}^{0}\left(\mathbb{R}^{N}\right)\right\},
$$

still denoted by $\tilde{F}$. Then, the functional defined by

$$
\varphi \in C_{0}^{0}\left(\mathbb{R}^{N}\right)^{N \times N} \longmapsto \tilde{F}[\varphi]
$$

is a continuous linear mapping which vanishes on the space $\left\{h I_{N}: h \in C_{0}^{0}\left(\mathbb{R}^{N}\right)\right\}$. Hence, there exists $\mu \in$ $\mathcal{M}\left(\mathbb{R}^{N}\right)^{N \times N}$, with $\operatorname{tr}(\mu)=0$, such that

$$
\langle\tilde{F}, u\rangle=\int_{\mathbb{R}^{N}} u: \mathrm{d} \mu, \quad \forall u \in C_{0}^{0}\left(\mathbb{R}^{N}\right)^{N \times N},
$$

which, by construction of $\tilde{F}$, implies that $F=-\operatorname{Div}(\mu)$. Now, applying the first part of the proof and taking into account that $\operatorname{tr}(\mu)=0$, we get that $q=0$, which concludes the proof of Theorem 2.2. 
Proof of Corollary 2.3. It is enough to remark that the equality $\nabla g=\operatorname{Div}(\mu)$ implies that the distribution $F=\operatorname{Div}(\mu)$ satisfies the conditions of Theorem 2.2. The proof of the theorem then shows that there exists $p \in \hat{W}^{-1, N^{\prime}}\left(\mathbb{R}^{N}\right)$ such that

$$
\nabla\left(p+\frac{1}{N} \operatorname{tr}(\mu)\right)=\operatorname{Div}(\mu) \text { in } \mathcal{D}^{\prime}\left(\mathbb{R}^{N}\right), \quad \text { with } \quad\|p\|_{\hat{W}^{-1, N^{\prime}}\left(\mathbb{R}^{N}\right)} \leq C\|\mu\|_{\mathcal{M}\left(\mathbb{R}^{M}\right)^{N \times N}} .
$$

On the other hand, the equality

$$
\nabla\left(p+\frac{1}{N} \operatorname{tr}(\mu)\right)=\nabla g \quad \text { in } \mathcal{D}^{\prime}\left(\mathbb{R}^{N}\right)^{N}
$$

implies the existence of a constant $R \in \mathbb{R}$ such that

$$
p+\frac{1}{N} \operatorname{tr}(\mu)=g+R
$$

From (2.27) and (2.28) we deduce (2.8).

Remark 2.6. Lemma 2.5 proves in particular that a distribution $F \in \hat{W}^{-1, r}\left(\mathbb{R}^{N}\right)^{N}$ such that $\operatorname{Curl}(F)=0$ in $\mathcal{D}^{\prime}\left(\mathbb{R}^{N}\right)^{N \times N}$ (this holds if $F$ satisfies (2.3)) is the gradient of a function in $L^{r}\left(\mathbb{R}^{N}\right)$. This classical representation permits to estimate the pressure in fluid mechanics. Theorem 2.2 extends this result to the case $r=1$. The proof of Lemma 2.5 below shows how the classical result is derived from the Calderón-Zygmund estimate, which does not hold for $r=1$. Some of the computations of this proof will be used in the next section.

Proof of Lemma 2.5. Let $F$ be a distribution in $\hat{W}^{-k, r}\left(\mathbb{R}^{N}\right)^{N}$. By definition (see Sect. 1) $F$ read as

$$
F=\sum_{I \in\{1, \ldots, N\}^{k}} \partial_{I}^{k} f_{I}, \quad \text { with } f_{I} \in L^{r}\left(\mathbb{R}^{N}\right)^{N}
$$

Consider a sequence of functions $\varphi^{R} \in C_{c}^{\infty}\left(\mathbb{R}^{N}\right)$ such that $\varphi^{R}=1$ in $B(0, R), \varphi^{R}=0$ outside $B(0,2 R)$ and

$$
\left\|D^{j} \varphi^{R}\right\|_{L^{\infty}\left(\mathbb{R}^{N}\right)^{N^{j}}} \leq \frac{C_{j}}{R^{j}}, \quad \forall j \geq 0, \forall R>1 .
$$

Then, the distribution $F^{R}$ defined by

$$
F^{R}:=\sum_{I \in\{1, \ldots, N\}^{k}} \partial_{I}^{k}\left(\varphi^{R} f_{I}\right)
$$

has support in $B(0,2 R)$ and strongly converges to $F$ in $\hat{W}^{-k, r}\left(\mathbb{R}^{N}\right)^{N}$ as $R$ tends to infinity.

Define the distribution $p^{R}$ by

$$
p^{R}:=\Gamma * \operatorname{div}\left(F^{R}\right)=\sum_{j=1}^{N} \partial_{j}\left(\Gamma * F_{j}^{R}\right),
$$

where $\Gamma$ is the fundamental solution of the Laplace operator defined by (1.5). Then, since $\Delta\left(\Gamma * F^{R}\right)=F^{R}$, the vector-valued distribution $G^{R}:=F^{R}-\nabla p^{R}$ satisfies

$$
\begin{aligned}
G_{i}^{R} & =\sum_{j=1}^{N}\left(\partial_{j j}^{2}\left(\Gamma * F_{i}^{R}\right)-\partial_{i j}^{2}\left(\Gamma * F_{j}^{R}\right)\right) \\
& =-\sum_{j=1}^{N} \partial_{j} \Gamma *\left(\operatorname{Curl}\left(F^{R}\right)\right)_{i j}, \quad 1 \leq i \leq N .
\end{aligned}
$$


Hence, by the Calderón-Zygmund estimates (1.8) we get

$$
\left\{\begin{aligned}
\left\|p^{R}\right\|_{\hat{W}^{-k+1, r}\left(\mathbb{R}^{N}\right)^{N}} & \leq C\left\|\operatorname{div}\left(F^{R}\right)\right\|_{\hat{W}^{-k-1, r}\left(\mathbb{R}^{N}\right)} \\
\left\|G^{R}\right\|_{\hat{W}^{-k, r}\left(\mathbb{R}^{N}\right)^{N}} & \leq C\left\|\operatorname{Curl}\left(F^{R}\right)\right\|_{\hat{W}^{-k-1, r}\left(\mathbb{R}^{N}\right)^{N \times N}} .
\end{aligned}\right.
$$

Moreover, by linearity the strong convergence of $F^{R}$ to $F$ in $\hat{W}^{-k, r}\left(\mathbb{R}^{N}\right)^{N}$ ensures the existence of the limit $p$ of $p^{R}$ in $\hat{W}^{-k+1, r}\left(\mathbb{R}^{N}\right)$ and the limit $G$ of $G^{R}$ in $\hat{W}^{-k, r}\left(\mathbb{R}^{N}\right)^{N}$, which satisfy (2.24) and (2.26). Finally, it is easy to deduce from the first equality of (2.30) that $G^{R}$ is divergence free, and hence that $G$ satisfies (2.25).

\subsection{The case of a bounded open set}

In this section we consider the case of a bounded open subset $\Omega$ of $\mathbb{R}^{N}$. Thanks to the results of the previous section we will prove local and global estimates for a measure $\mu \in \mathcal{M}(\Omega)^{N \times N}$ satisfying $\operatorname{Curl}(\operatorname{Div}(\mu))=0$ in $\mathcal{D}^{\prime}(\Omega)^{N \times N}$. The corresponding results are given by the following theorems:

Theorem 2.7. Consider two bounded open sets $\omega, \Omega$ of $\mathbb{R}^{N}$, such that $\bar{\omega} \subset \Omega$. Then, there exists a constant $C>0$ which only depends on $\omega$ and $\Omega$, such that any matrix-valued measure $\mu \in \mathcal{M}(\Omega)^{N \times N}$, with $\operatorname{Curl}(\operatorname{Div}(\mu))=0$ in $\mathcal{D}^{\prime}(\Omega)^{N \times N}$, satisfies

$$
\mu-\frac{1}{N} \operatorname{tr}(\mu) I_{N} \in W^{-1, N^{\prime}}(\omega)^{N \times N}
$$

with

$$
\left\|\mu-\frac{1}{N} \operatorname{tr}(\mu) I_{N}\right\|_{W^{-1, N^{\prime}}(\omega)^{N \times N}} \leq C\|\mu\|_{\mathcal{M}(\Omega)^{N \times N}} .
$$

Theorem 2.8. Consider a bounded open set $\Omega$ of $\mathbb{R}^{N}$, of class $C^{3}$. Then, there exists a constant $C>0$ such that any matrix-valued measure $\mu \in \mathcal{M}(\Omega)^{N \times N}$, with $\operatorname{Curl}(\operatorname{Div}(\mu))=0$ in $\mathcal{D}^{\prime}(\Omega)^{N \times N}$, satisfies

$$
\mu-\frac{1}{N} \operatorname{tr}(\mu) I_{N} \in W^{-1, N^{\prime}}(\Omega)^{N \times N}
$$

with

$$
\left\|\mu-\frac{1}{N} \operatorname{tr}(\mu) I_{N}\right\|_{W^{-1, N^{\prime}}(\Omega)^{N \times N}} \leq C\|\mu\|_{\mathcal{M}(\Omega)^{N \times N}}
$$

Theorems 2.7 and 2.8 imply the following local versions of Theorem 2.2 and Corollary 2.3, which provide estimates for a distribution whose gradient is the divergence of a matrix-valued measure. For the sake of simplicity we just state the global estimates which hold for a smooth open set. Local estimates are quite similar. Since the proofs of the corresponding results follow the same ideas as the ones of the whole space case, we do not give them. In particular, we use the classical result of Theorem 1.1 for $r=N^{\prime}$, which can be also deduced from Lemma 2.5.

Theorem 2.9. Let $\Omega$ be a bounded connected open subset of $\mathbb{R}^{N}$, of class $C^{3}$. Consider a distribution $F$ in $\mathcal{D}^{\prime}(\Omega)^{N}$ such that

$$
\langle F, \varphi\rangle=0, \quad \forall \varphi \in C_{c}^{\infty}(\Omega)^{N}, \text { with } \operatorname{div}(\varphi)=0,
$$

and such that there exists a constant $M>0$ satisfying

$$
\langle F, \varphi\rangle \leq M\|D \varphi\|_{L^{\infty}(\Omega)^{N \times N}}, \quad \forall \varphi \in C_{c}^{\infty}(\Omega)^{N}
$$

Then, there exist a distribution $p \in W^{-1, N^{\prime}}(\Omega)$, a measure $q \in \mathcal{M}(\Omega)$, and a constant $C>0$ such that

$$
\begin{gathered}
F=\nabla(p+q) \quad \text { in } \mathcal{D}^{\prime}(\Omega)^{N}, \\
\|p\|_{W^{-1, N^{\prime}}(\Omega)}+\|q\|_{\mathcal{M}(\Omega)} \leq C M .
\end{gathered}
$$


Moreover, if $F$ satisfies

$$
\langle F, \varphi\rangle \leq M \inf \left\{\|D \varphi-h I\|_{L^{\infty}(\Omega)}: h \in C_{0}^{0}(\Omega)\right\}, \quad \forall \varphi \in C_{c}^{\infty}(\Omega),
$$

then we can take $q=0$.

Corollary 2.10. Let $\Omega$ be a bounded connected open subset of $\mathbb{R}^{N}$, of class $C^{3}$. There exists $C>0$ such that for any $g \in \mathcal{D}^{\prime}(\Omega)$ satisfying $\nabla g=\operatorname{Div}(\mu)$ in $\mathcal{D}^{\prime}(\Omega)^{N}$, with $\mu \in \mathcal{M}(\Omega)^{N \times N}$, there exists a constant $R \in \mathbb{R}$ such that

$$
g-\frac{1}{N} \operatorname{tr}(\mu)-R \in W^{-1, N^{\prime}}(\Omega), \quad \text { with } \quad\left\|g-\frac{1}{N} \operatorname{tr}(\mu)-R\right\|_{W^{-1, N^{\prime}}(\Omega)} \leq C\|\mu\|_{\mathcal{M}(\Omega)^{N \times N}} .
$$

Proof of Theorem 2.7. Along the proof we denote by $C>0$ a generic constant which can change from line to line and which only depends on $\omega$ and $\Omega$.

Take two functions $\Phi, \Psi \in C_{c}^{\infty}(\Omega)$ such that

$$
\Phi=\Psi=1 \text { in } \omega, \quad \int_{\Omega} \Phi \mathrm{d} x \neq 0, \quad \int_{\Omega} \Psi \mathrm{d} x=0
$$

Let $\mu \in \mathcal{M}(\Omega)^{N \times N}$ under the assumptions of the theorem, and set

$$
\bar{\mu}:=\frac{\int_{\Omega} \Phi \mathrm{d} \mu}{\int_{\Omega} \Phi \mathrm{d} x} \text { and } \tilde{\mu}:=\Phi(\mu-\bar{\mu})+\Psi \bar{\mu} .
$$

We can assume that the matrix-valued measure $\tilde{\mu}$ is defined in the whole space $\mathbb{R}^{N}$, by taking $\tilde{\mu}=0$ in $\mathbb{R}^{N} \backslash \Omega$. Then, we have

$$
|\tilde{\mu}|\left(\mathbb{R}^{N} \backslash \Omega\right)=0, \quad \tilde{\mu}=\mu \text { in } \omega, \quad\|\tilde{\mu}\|_{\mathcal{M}\left(\mathbb{R}^{N}\right)^{N \times N}} \leq C\|\mu\|_{\mathcal{M}(\Omega)^{N \times N}}, \quad \tilde{\mu}(\Omega)=0 .
$$

Moreover, taking into account that $\operatorname{Curl}(\operatorname{Div}(\mu))=0$, a direct computation yields

$$
\operatorname{Curl}(\operatorname{Div}(\tilde{\mu}))=\operatorname{Curl}((\mu-\bar{\mu}) \nabla \Phi)+\nabla \Phi \otimes \operatorname{Div}(\mu)-\operatorname{Div}(\mu) \otimes \nabla \Phi+\nabla^{2} \Psi \bar{\mu}^{T}-\bar{\mu} \nabla^{2} \Psi
$$

Since $\mathcal{M}(\Omega)$ is continuously embedded in $W^{-1, r}(\Omega)$ for $r \in(1, N)$, we deduce from $(2.42)$ that $\operatorname{Curl}(\operatorname{Div}(\tilde{\mu}))$ belongs to $W^{-2, r}\left(\mathbb{R}^{N}\right)^{N \times N}$. More precisely, thanks to $(2.41)$ it is easy to check that there exists a constant $C>0$ such that for any $\varphi \in W^{2, r^{\prime}}\left(\mathbb{R}^{N}\right)^{N \times N}$,

$$
\begin{aligned}
\langle\operatorname{Curl}(\operatorname{Div}(\tilde{\mu})), \varphi\rangle & \leq C\|\mu\|_{\mathcal{M}(\Omega)^{N \times N}}\|\varphi\|_{W^{2, r^{\prime}}(\Omega)^{N \times N}} \\
& \leq C\|\mu\|_{\mathcal{M}(\Omega)^{N \times N}}\left(\|\varphi\|_{L^{r^{\prime}}(\Omega)^{N \times N}}+\left\|D^{2} \varphi\right\|_{L^{r^{\prime}}(\Omega)^{N^{4}}}\right)
\end{aligned}
$$

This implies that

$$
\operatorname{Curl}(\operatorname{Div}(\tilde{\mu}))=g+h, \quad \text { with } \begin{cases}g \in L^{r}\left(\mathbb{R}^{N}\right)^{N \times N} & \operatorname{supp}(g) \subset \bar{\Omega} \subset U \\ h \in \hat{W}^{-2, r}\left(\mathbb{R}^{N}\right)^{N \times N} & \operatorname{supp}(h) \subset \bar{\Omega} \subset U\end{cases}
$$

where $U$ is a ball in $\mathbb{R}^{N}$ centered at the origin such that $\bar{\Omega} \subset U$, and the norms of $g$ and $h$ are bounded by $\|\mu\|_{\mathcal{M}(\Omega)^{N \times N}}$. 
Now, using that $\Gamma$ is the fundamental solution of the Laplace operator we can check that the distribution $p$ and the vector-valued distribution $u$ defined by

$$
\begin{gathered}
p:=\sum_{j=1}^{N} \partial_{j} \Gamma *(\operatorname{Div}(\tilde{\mu}))_{j}=\sum_{j, k=1}^{N} \partial_{j k}^{2} \Gamma * \tilde{\mu}_{j k}, \quad \text { and } \\
u_{i}:=\sum_{j=1}^{N} \partial_{j}(\Gamma * \Gamma) *(\operatorname{Curl}(\operatorname{Div}(\tilde{\mu})))_{i j}, \quad 1 \leq i \leq N,
\end{gathered}
$$

are solutions of the Stokes problem

$$
\left\{\begin{aligned}
-\Delta u+\nabla p=\operatorname{Div}(\tilde{\mu}) & \text { in } \mathbb{R}^{N} \\
\operatorname{div}(u)=0 & \text { in } \mathbb{R}^{N} .
\end{aligned}\right.
$$

Taking the Curl operator in (2.46) we get that $D u$ satisfies

$$
\operatorname{Curl}(\operatorname{Div}(D u+\tilde{\mu}))=0 \quad \text { in } \mathcal{D}^{\prime}\left(\mathbb{R}^{N}\right)^{N \times N} .
$$

Moreover, we deduce from (2.45) that

$$
\partial_{k} u_{i}=\sum_{j=1}^{N} \partial_{k j}^{2}(\Gamma * \Gamma) *(\operatorname{Curl}(\operatorname{Div}(\tilde{\mu})))_{i j} .
$$

Therefore, by the inequality (2.43) combined with the Calderón-Zygmund estimates (1.6) (for $g$ ) and (1.8) (for $h$ ), we obtain that $D u$ belongs to $L^{r}(U)^{N \times N}$, with

$$
\|D u\|_{L^{r}(U)^{N \times N}} \leq C\|\mu\|_{\mathcal{M}(\Omega)^{N \times N}} .
$$

On the other hand, since $\Gamma$ is a radial function, so is $\Gamma * \Gamma$. Then, using the definition (1.5) of $\Gamma$ and the Laplace operator in cylindrical coordinates, we can solve the equation $\Delta(\Gamma * \Gamma)=\Gamma$ in $\mathbb{R}^{N}$ as a first-order ordinary differential equation of the variable $|x|$, which leads us to the following explicit formula for $\Gamma * \Gamma$ :

$$
(\Gamma * \Gamma)(x)=\left\{\begin{array}{cl}
\frac{1}{2 N(N-2)(N-4)|B(0,1)||x|^{N-4}} & \text { if } N \neq 2,4 \\
\frac{|x|^{2}}{8 \pi}(\log |x|-1) & \text { if } N=2 \\
\frac{-1}{16|B(0,1)|} \log |x| & \text { if } N=4 .
\end{array}\right.
$$

The expression (2.50) of $\Gamma * \Gamma$ allows us to derive the inequality

$$
\left|D^{5}(\Gamma * \Gamma)(x)\right| \leq\left\{\begin{array}{ll}
\frac{C}{|x|^{N+1}} & \text { if } N>2 \\
\frac{C}{|x|^{3}}(1+\log |x|) & \text { if } N=2,
\end{array} \quad \text { for } x \in \mathbb{R}^{N} \backslash\{0\} .\right.
$$


This combined with the first, third and fourth assertions in (2.41), implies that for any $x \notin U$,

$$
\begin{aligned}
\left|\left(\partial_{i j k l}^{4}(\Gamma * \Gamma) * \tilde{\mu}_{m n}\right)(x)\right| & =\left|\int_{\Omega}\left(\partial_{i j k l}^{4}(\Gamma * \Gamma)(x-y)-\partial_{i j k l}^{4}(\Gamma * \Gamma)(x)\right) \tilde{\mu}_{m n}(y) \mathrm{d} y\right| \\
& \leq \begin{cases}\frac{C\|\mu\|_{\mathcal{M}(\Omega)^{N \times N}}}{(1+|x|)^{N+1}} & \text { if } N>2 \\
\frac{C\|\mu\|_{\mathcal{M}(\Omega)^{N \times N}}}{(1+|x|)^{3}} \log (2+|x|) & \text { if } N=2 .\end{cases}
\end{aligned}
$$

Therefore, by (2.49) and by applying (2.52) to the expression (2.48) of $D u$, we get that $D u$ belongs to $L^{1}\left(\mathbb{R}^{N}\right)^{N \times N}$, and

$$
\|D u\|_{L^{1}\left(\mathbb{R}^{N}\right)^{N \times N}} \leq C\|\mu\|_{\mathcal{M}(\Omega)^{N \times N}}
$$

By virtue of (2.47), the third assertion in (2.41) and (2.53), we can apply Theorem 2.1, which gives

$$
D u+\tilde{\mu}-\frac{1}{N} \operatorname{tr}(D u+\tilde{\mu}) I_{N} \in \hat{W}^{-1, N^{\prime}}\left(\mathbb{R}^{N}\right)^{N \times N},
$$

with

$$
\left\|D u+\tilde{\mu}-\frac{1}{N} \operatorname{tr}(D u+\tilde{\mu}) I_{N}\right\|_{\hat{W}^{-1, N^{\prime}}\left(\mathbb{R}^{N}\right)^{N \times N}} \leq C\|\mu\|_{\mathcal{M}(\Omega)^{N \times N}} .
$$

This combined with $\tilde{\mu}=\mu$ in $\omega,(2.49)$ and the inequality (recall that $1<r<N^{\prime}$ )

$$
\|D u\|_{\hat{W}^{-1, N^{\prime}}(\Omega)^{N \times N}} \leq C\|D u\|_{L^{r}(\Omega)^{N \times N}},
$$

yields finally (2.32).

Proof of Theorem 2.8. It is a simple consequence of Theorem 2.7 and the following result which provides estimates near the boundary:

Lemma 2.11. Let $\Omega$ a bounded open set of $\mathbb{R}^{N}$ and let $x_{0} \in \partial \Omega$. Assume that there exist $r>0$, an open set $\Theta$ of $\mathbb{R}^{N}$, with $x_{0} \in \Theta$, and a $C^{3}$-diffeomorphism $\psi$ from a neighborhood of $\bar{B}(0, r)$ onto an neighborhood of $\bar{\Theta}$ such that

$$
\psi(0)=x_{0}, \quad \psi(B(0, r))=\Theta, \quad \psi\left(B(0, r)^{+}\right)=\Theta \cap \Omega \stackrel{\text { def }}{=} \Theta^{+},
$$

where $B(0, r)^{+}$denotes the upper half ball. Then, denoting $\tilde{\Theta}^{+}:=\psi\left(B(0, r / 2)^{+}\right)$, there exists a constant $C>0$ such that for any $\mu$ in $\mathcal{M}\left(\Theta^{+}\right)^{N \times N}$, with $\operatorname{Curl}(\operatorname{Div}(\mu))=0$ in $\Theta^{+}$, we have

$$
\mu-\frac{1}{N} \operatorname{tr}(\mu) I_{N} \in W^{-1, N^{\prime}}\left(\tilde{\Theta}^{+}\right), \quad \text { with } \quad\left\|\mu-\frac{1}{N} \operatorname{tr}(\mu) I_{N}\right\|_{W^{-1, N^{\prime}\left(\tilde{\Theta}^{+}\right)}} \leq C\|\mu\|_{\mathcal{M}\left(\Theta^{+}\right)^{N}} .
$$

The proof of Lemma 2.11 which is rather technical is given in Appendix A.2.

\section{The Navier-Stokes equation with a Viscosity in $L^{1}$}

In the section we consider a $C^{3}$-regular bounded connected open subset $\Omega$ of $\mathbb{R}^{N}$, and a fourth-order tensorvalued function $A \in L^{1}(\Omega)^{N^{4}}$ such that for a.e. $x \in \Omega, A(x)$ maps the set of symmetric matrices $\mathbb{R}_{s}^{N \times N}$ into itself, it is symmetric, $\alpha$-coercive for a given $\alpha>0$, and preserves the set of zero trace matrices, i.e.

$$
\forall \xi, \eta \in \mathbb{R}_{s}^{N \times N},\left\{\begin{array}{l}
A(x) \xi: \eta=A(x) \eta: \xi \\
A(x) \xi: \xi \geq \alpha|\xi|^{2},
\end{array} \quad \operatorname{tr}(\xi)=0 \Rightarrow \operatorname{tr}(A(x) \xi)=0, \quad \text { a.e. } x \in \Omega .\right.
$$


Then, we have the following result:

Theorem 3.1. Let $f \in H^{-1}(\Omega)^{N}, N \leq 4$. Then, there exist a velocity $u \in H_{0}^{1}(\Omega)^{N}$, with

$$
\int_{\Omega} A E(u): E(u) \mathrm{d} x<\infty, \quad A E(u) \in W^{-1, N^{\prime}}(\Omega)^{N \times N} \cap L^{1}(\Omega)^{N \times N},
$$

and a pressure $p \in W^{-1, N^{\prime}}(\Omega) / \mathbb{R}$ (defined up to an additive constant) solutions of the Navier-Stokes equation in the distributions sense

$$
\begin{cases}-\operatorname{Div}(A E(u))+(u \cdot \nabla) u+\nabla p=f & \text { in } \Omega \\ \operatorname{div}(u)=0 & \text { in } \Omega .\end{cases}
$$

Moreover, there exists a constant $C_{\Omega}>0$ only depending on $\Omega$, such that the pressure $p$ and the viscosity term $A E(u)$ satisfy the estimate

$$
\|p\|_{W^{-1, N^{\prime}}(\Omega) / \mathbb{R}}+\|A E(u)\|_{W^{-1, N^{\prime}}(\Omega)^{N \times N}} \leq C_{\Omega}\|f\|_{H^{-1}(\Omega)^{N}}\left(\alpha^{-\frac{1}{2}}\|A\|_{L^{1}(\Omega)^{N^{4}}}^{\frac{1}{2}}+\alpha^{-2}\|f\|_{H^{-1}(\Omega)^{N}}+1\right) .
$$

Proof of Theorem 3.1. The proof is divided into three steps.

First step: Existence of the velocity. Let $H$ be the functional space defined by

$$
H:=\left\{v \in H_{0}^{1}(\Omega)^{N}: \operatorname{div}(v)=0 \text { in } \mathcal{D}^{\prime}(\Omega) \text { and } \int_{\Omega} A E(v): E(v) \mathrm{d} x<\infty\right\}
$$

endowed with the norm

$$
\|v\|_{H}:=\left(\int_{\Omega} A E(v): E(v) \mathrm{d} x\right)^{\frac{1}{2}}, \quad \text { for } v \in H .
$$

Let us prove that $\left(H,\|\cdot\|_{H}\right)$ is a Hilbert space. It is enough to check that $H$ is complete for its norm. Let $v_{n}$ be a Cauchy sequence in $\left(H,\|\cdot\|_{H}\right)$. Then, for any $\varepsilon>0$, there exists $N_{\varepsilon} \in \mathbb{N}$ such that

$$
\forall m, n \geq N_{\varepsilon}, \quad \int_{\Omega} A\left(E\left(v_{m}\right)-E\left(v_{n}\right)\right):\left(E\left(v_{m}\right)-E\left(v_{n}\right)\right) \mathrm{d} x<\varepsilon .
$$

Thanks to the $\alpha$-coercivity of $A$ (3.1) combined with the Korn inequality the sequence $v_{n}$ strongly converges to some divergence free function $v$ in $H_{0}^{1}(\Omega)^{N}$. This implies in particular that for any fixed $m \in \mathbb{N}$, there exists a subsequence of $n$ still denoted by $n$, such that $A\left(E\left(v_{m}\right)-E\left(v_{n}\right)\right):\left(E\left(v_{m}\right)-E\left(v_{n}\right)\right)$ converges to $A\left(E\left(v_{m}\right)-E(v)\right):\left(E\left(v_{m}\right)-E(v)\right)$ a.e. in $\Omega$. Since this sequence is nonnegative, from Fatou's lemma we deduce that

$$
\begin{aligned}
& \int_{\Omega} A\left(E\left(v_{m}\right)-E(v)\right):\left(E\left(v_{m}\right)-E(v)\right) \mathrm{d} x \\
& \leq \liminf _{n \rightarrow \infty} \int_{\Omega} A\left(E\left(v_{m}\right)-E\left(v_{n}\right)\right):\left(E\left(v_{m}\right)-E\left(v_{n}\right)\right) \mathrm{d} x \leq \varepsilon, \quad \forall m \geq N_{\varepsilon} .
\end{aligned}
$$

Due to the arbitrariness of $\varepsilon$ estimate (3.8) implies that both $v \in H$ and the sequence $v_{n}$ converges to $v$ in $H$.

Since the set of divergence free functions in $C_{c}^{\infty}(\Omega)^{N}$ is clearly contained in the space $H$, the closure $V$ of this set in $H$ :

$$
V:={\overline{\left\{v \in C_{c}^{\infty}(\Omega)^{N}: \operatorname{div}(v)=0 \text { in } \mathcal{D}^{\prime}(\Omega)\right.}}^{H},
$$

also defines a Hilbert space. 
Now, replacing the usual space of divergence free functions in $H_{0}^{1}(\Omega)^{N}$ by the new Hilbert space $V(3.9)$, we can easily repeat the classical construction of a solution of the Navier-Stokes equation thanks to the Galerkin method (see $[12,14,15,20,21])$ to obtain a velocity $u \in V$ satisfying the variational formulation

$$
\int_{\Omega}(A E(u): E(\varphi)+(u \cdot \nabla) u \cdot \varphi) \mathrm{d} x=\langle f, \varphi\rangle_{H^{-1}(\Omega)^{N}, H_{0}^{1}(\Omega)^{N}}, \quad \forall \varphi \in V .
$$

More precisely, the function $u$ is the weak limit in $H$ of a divergence free sequence $u_{n}$ in $C_{c}^{\infty}(\Omega)^{N}$ which by virtue of the Galerkin construction satisfies

$$
\int_{\Omega} A E\left(u_{n}\right): E\left(u_{n}\right) \mathrm{d} x=\left\langle f, u_{n}\right\rangle_{H^{-1}(\Omega)^{N}, H_{0}^{1}(\Omega)^{N}} .
$$

This combined with the lower semi-continuity of the Hilbert norm $\|\cdot\|_{H}$ in $H$, yields

$$
\int_{\Omega} A E(u): E(u) \mathrm{d} x \leq\langle f, u\rangle_{H^{-1}(\Omega)^{N}, H_{0}^{1}(\Omega)^{N}} .
$$

Moreover, since the symmetric bilinear form

$$
(\xi, \eta) \in\left(\mathbb{R}_{s}^{2 \times 2}\right)^{2} \longmapsto A \xi: \eta
$$

is associated with a nonnegative quadratic form, the Cauchy-Schwarz inequality implies that for any symmetric matrix $\xi \in \mathbb{R}_{s}^{2 \times 2}$, with $|\xi|=1$,

$$
|A E(u): \xi| \leq(A \xi: \xi)^{\frac{1}{2}}(A E(u): E(u))^{\frac{1}{2}} \quad \text { a.e. in } \Omega \text {. }
$$

This combined with the inequality $A \xi: \xi \leq|A||\xi|^{2}$, yields

$$
|A E(u)|=\sup _{\left\{\xi \in \mathbb{R}_{s}^{2 \times 2}:|\xi|=1\right\}}|A E(u): \xi| \leq|A|^{\frac{1}{2}}(A E(u): E(u))^{\frac{1}{2}} \quad \text { a.e. in } \Omega \text {. }
$$

Now, using the Cauchy-Schwarz inequality for the integral and (3.11) we get

$$
\int_{\Omega}|A E(u)| \mathrm{d} x \leq\left(\int_{\Omega}|A| \mathrm{d} x\right)^{\frac{1}{2}}\left(\int_{\Omega} A E(u): E(u) \mathrm{d} x\right)^{\frac{1}{2}}<\infty
$$

hence $A E(u) \in L^{1}(\Omega)^{N \times N}$.

Second step: Estimate of the pressure. We take $z \in H_{0}^{1}(\Omega)$ solution of $\Delta z=f$ in $\Omega$. By Theorem 2.9 and $\operatorname{div}(u)=0$ in $\Omega$, there exists $r \in W^{-1, N^{\prime}}(\Omega)+\mathcal{M}(\Omega)$ such that

$$
\operatorname{Div}(A E(u)-u \otimes u+D z)=\operatorname{Div}(A E(u))-(u \cdot \nabla) u+f=\nabla r \quad \text { in } \mathcal{D}^{\prime}(\Omega)^{N}
$$

Moreover, since the assumptions on $A$ and the fact that $\operatorname{div}(u)=0$ imply that $\operatorname{tr}(A E(u))=0$, we can apply Corollary 2.10 to deduce the existence of a constant $c>0$ only depending on $\Omega$ and a constant $R>0$ such that

$$
\left\|r+\frac{1}{N}\left(|u|^{2}-\operatorname{div}(z)\right)-R\right\|_{W^{-1, N^{\prime}}(\Omega)} \leq c\|A E(u)-u \otimes u+D z\|_{L^{1}(\Omega)^{N \times N}} .
$$

On the other hand, by (3.11), (3.12), the $\alpha$-coerciveness of $A$, the Korn inequality, and the Sobolev embedding of $L^{2}(\Omega)$ into $W^{-1, N^{\prime}}(\Omega)$ and of $H_{0}^{1}(\Omega)$ into $L^{4}(\Omega)$ (recall that $N \leq 4$ ), we have for another constant $c$ 
which only depends on $\Omega$,

$$
\begin{gathered}
\|\operatorname{div}(z)\|_{W^{-1, N^{\prime}}(\Omega)}+\|D z\|_{L^{1}(\Omega)^{N \times N}} \leq c\|f\|_{H^{-1}(\Omega)^{N}}, \\
\left\||u|^{2}\right\|_{W^{-1, N^{\prime}}(\Omega)}+\|u \otimes u\|_{L^{2}(\Omega)^{N \times N}} \leq c \alpha^{-2}\|f\|_{H^{-1}(\Omega)^{N}}^{2}, \\
\|A E(u)\|_{L^{1}(\Omega)^{N \times N}} \leq c \alpha^{-\frac{1}{2}}\|A\|_{L^{1}(\Omega)^{N^{4}}}^{\frac{1}{2}}\|f\|_{H^{-1}(\Omega)^{N}} .
\end{gathered}
$$

Then, taking $p:=r-R$, we get that (3.3) is satisfied and that

$$
\|p\|_{W^{-1, N^{\prime}}(\Omega)} \leq C_{\Omega}\|f\|_{H^{-1}(\Omega)^{N}}\left(\alpha^{-\frac{1}{2}}\|A\|_{L^{1}(\Omega)^{N^{4}}}^{\frac{1}{2}}+\alpha^{-2}\|f\|_{H^{-1}(\Omega)^{N}}+1\right) .
$$

Third step: Estimate of $A E(u)$. A straightforward consequence of (3.13) is that

$$
\operatorname{Curl}(\operatorname{Div}(A E(u)-u \otimes u+D z))=0 \quad \text { in } \mathcal{D}^{\prime}(\Omega)^{N \times N} .
$$

Therefore, by virtue of Theorem 2.8 combined with estimates (3.15)-(3.18) we obtain (3.4), which concludes the proof.

\section{A. Appendix}

\section{A.1. Proof of Lemma 2.4}

The proof is based on the one of Theorem 1.5 in [23] (see also [22] for related results).

Using a regularization argument we can assume that $g=\left(g_{1}, \ldots, g_{N}\right) \in C_{c}^{\infty}\left(\mathbb{R}^{N}\right)^{N \times N}$, where $g_{j}$ denotes the $j$-th column of the matrix-valued $g$.

Let $u=\left(u_{1}, \ldots, u_{N}\right) \in C_{c}^{\infty}\left(\mathbb{R}^{N}\right)^{N}$. We have

$$
\int_{\mathbb{R}^{N}} f_{1}(x) u_{1}(x) \mathrm{d} x=\int_{\mathbb{R}} \int_{\mathbb{R}^{N-1}} f_{1}\left(x_{1}, x^{\prime}\right) u_{1}\left(x_{1}, x^{\prime}\right) \mathrm{d} x^{\prime} \mathrm{d} x_{1} .
$$

Consider $\rho \in C_{c}^{\infty}\left(B(0,1) \cap \mathbb{R}^{N-1}\right)$, and define the mollifier $\rho_{\varepsilon}$ in $\mathbb{R}^{N-1}$ by

$$
\rho_{\varepsilon}\left(x^{\prime}\right)=\varepsilon^{1-N} \rho\left(\frac{x^{\prime}}{\varepsilon}\right), \quad \text { for } x^{\prime}=\left(x_{2}, \ldots, x_{N}\right) \in \mathbb{R}^{N-1},
$$

where the parameter $\varepsilon>0$ will be chosen later as a function of the variable $x_{1}$.

We have for a fixed $x_{1} \in \mathbb{R}$,

$$
\int_{\mathbb{R}^{N-1}} f_{1}\left(x_{1}, x^{\prime}\right) u_{1}\left(x_{1}, x^{\prime}\right) \mathrm{d} x^{\prime}=A_{1}+A_{2}
$$

where

$$
\begin{aligned}
& A_{1}:=\int_{\mathbb{R}^{N-1}} f_{1}\left(x_{1}, x^{\prime}\right)\left(\rho_{\varepsilon} * u_{1}\right)\left(x_{1}, x^{\prime}\right) \mathrm{d} x^{\prime}, \\
& A_{2}:=\int_{\mathbb{R}^{N-1}} f_{1}\left(x_{1}, x^{\prime}\right)\left(u_{1}\left(x_{1}, x^{\prime}\right)-\left(\rho_{\varepsilon} * u_{1}\right)\left(x_{1}, x^{\prime}\right)\right) \mathrm{d} x^{\prime} .
\end{aligned}
$$

In order to estimate $A_{1}$, note that for any $x_{1} \in \mathbb{R}$, we have

$$
0=\sum_{j=2}^{N} \int_{\mathbb{R}^{N-1}} \int_{-\infty}^{x_{1}} \partial_{j}\left(g_{j}\left(t, x^{\prime}\right) \cdot\left(\rho_{\varepsilon} * \nabla_{x^{\prime}} u_{1}\right)\left(x_{1}, x^{\prime}\right)\right) \mathrm{d} x^{\prime}=B_{1}+B_{2}
$$


where

$$
\begin{aligned}
B_{1} & :=\sum_{j=2}^{N} \int_{\mathbb{R}^{N-1}} \int_{-\infty}^{x_{1}} \partial_{j} g_{j}\left(t, x^{\prime}\right) \cdot\left(\rho_{\varepsilon} * \nabla_{x^{\prime}} u_{1}\right)\left(x_{1}, x^{\prime}\right) \mathrm{d} x^{\prime}, \\
B_{2} & :=\sum_{j=2}^{N} \int_{\mathbb{R}^{N-1}} \int_{-\infty}^{x_{1}} g_{j}\left(t, x^{\prime}\right) \cdot \partial_{j}\left(\rho_{\varepsilon} * \nabla_{x^{\prime}} u_{1}\right)\left(x_{1}, x^{\prime}\right) \mathrm{d} x^{\prime} .
\end{aligned}
$$

Let us estimate $B_{1}$. Integrating by parts, and using that $f_{j}=\operatorname{div}\left(g_{j}\right)=\operatorname{div}_{x^{\prime}}\left(g_{j}\right)$, we have

$$
\begin{aligned}
B_{1} & =\sum_{j=2}^{N} \int_{\mathbb{R}^{N-1}} \int_{-\infty}^{x_{1}} \partial_{j} g_{j}\left(t, x^{\prime}\right) \cdot \nabla_{x^{\prime}}\left(\rho_{\varepsilon} * u_{1}\right)\left(x_{1}, x^{\prime}\right) \mathrm{d} x^{\prime} \\
& =-\sum_{j=2}^{N} \int_{\mathbb{R}^{N-1}} \int_{-\infty}^{x_{1}} \partial_{j}\left(\operatorname{div}_{x^{\prime}}\left(g_{j}\right)\right)\left(t, x^{\prime}\right)\left(\rho_{\varepsilon} * u_{1}\right)\left(x_{1}, x^{\prime}\right) \mathrm{d} x^{\prime} \\
& =-\sum_{j=2}^{N} \int_{\mathbb{R}^{N-1}} \int_{-\infty}^{x_{1}} \partial_{j} f_{j}\left(t, x^{\prime}\right)\left(\rho_{\varepsilon} * u_{1}\right)\left(x_{1}, x^{\prime}\right) \mathrm{d} x^{\prime},
\end{aligned}
$$

and since $f$ is divergence free, we get

$$
\begin{aligned}
B_{1} & =\int_{\mathbb{R}^{N-1}} \int_{-\infty}^{x_{1}} \partial_{1} f_{1}\left(t, x^{\prime}\right)\left(\rho_{\varepsilon} * u_{1}\right)\left(x_{1}, x^{\prime}\right) \mathrm{d} x^{\prime} \\
& =\int_{\mathbb{R}^{N-1}} f_{1}\left(x_{1}, x^{\prime}\right)\left(\rho_{\varepsilon} * u_{1}\right)\left(x_{1}, x^{\prime}\right) \mathrm{d} x^{\prime} .
\end{aligned}
$$

Let us now estimate $B_{2}$. By using the Hölder inequality in $\mathbb{R}^{N-1}$ with the exponents $N, N^{\prime}$, we have for any $j=2, \ldots, N$,

$$
\begin{aligned}
\mid \int_{\mathbb{R}^{N-1}} \int_{-\infty}^{x_{1}} g_{j}\left(t, x^{\prime}\right) \cdot \partial_{j} & \left(\rho_{\varepsilon} * \nabla_{x^{\prime}} u_{1}\right)\left(x_{1}, x^{\prime}\right) \mathrm{d} t \mathrm{~d} x^{\prime} \mid \\
\leq \int_{\mathbb{R}^{N-1}} \int_{-\infty}^{x_{1}}\left|g_{j}\left(t, x^{\prime}\right)\right| \mathrm{d} t \mathrm{~d} x^{\prime}\left\|\partial_{j}\left(\rho_{\varepsilon} * \nabla_{x^{\prime}} u_{1}\right)\left(x_{1}, \cdot\right)\right\|_{L^{\infty}\left(\mathbb{R}^{N-1}\right)^{N-1}} & \leq c \varepsilon^{\frac{1-N}{N}}\left\|g_{j}\right\|_{L^{1}\left(\mathbb{R}^{N}\right)^{N}}\left\|D_{x^{\prime}}^{2} u_{1}\left(x_{1}, \cdot\right)\right\|_{L^{N}\left(\mathbb{R}^{N-1}\right)^{(N-1) \times(N-1)}}
\end{aligned}
$$

This combined with (A.4) implies that

$$
\left|B_{2}\right| \leq c \varepsilon^{\frac{1-N}{N}} \sum_{j=2}^{N}\left\|g_{j}\right\|_{L^{1}\left(\mathbb{R}^{N}\right)^{N \times N}}\left\|D_{x^{\prime}}^{2} u_{1}\left(x_{1}, \cdot\right)\right\|_{L^{N}\left(\mathbb{R}^{N-1}\right)^{(N-1) \times(N-1)}} .
$$

Since $B_{1}+B_{2}=0$, and $A_{1}=B_{1}$ by (A.5), we deduce from (A.7) the following estimate for $A_{1}$

$$
\left|A_{1}\right| \leq\left|\int_{\mathbb{R}^{N-1}} f_{1}\left(x_{1}, x^{\prime}\right)\left(\rho_{\varepsilon} * u_{1}\right)\left(x_{1}, x^{\prime}\right) \mathrm{d} x^{\prime}\right| \leq c \varepsilon^{\frac{1-N}{N}}\|g\|_{L^{1}\left(\mathbb{R}^{N}\right)^{N \times N}}\left\|D_{x^{\prime}}^{2} u_{1}\left(x_{1}, \cdot\right)\right\|_{L^{N}\left(\mathbb{R}^{N-1}\right)^{(N-1) \times(N-1)}} .
$$


It remains to estimate $A_{2}$. To this end, by the Morrey-Sobolev embedding in $\mathbb{R}^{N-1}$ with Hölder exponent $1 / N$, we have

$$
\begin{aligned}
\left|A_{2}\right| & =\left|\int_{\mathbb{R}^{N-1}}\left(\operatorname{div}_{x^{\prime}}\left(g_{1}\right)\right)\left(x_{1}, x^{\prime}\right)\left(u_{1}\left(x_{1}, x^{\prime}\right)-\left(\rho_{\varepsilon} * u_{1}\right)\left(x_{1}, x^{\prime}\right)\right) \mathrm{d} x^{\prime}\right| \\
& =\left|\int_{\mathbb{R}^{N-1}} g_{1}\left(x_{1}, x^{\prime}\right) \cdot\left(\nabla_{x^{\prime}} u_{1}\left(x_{1}, x^{\prime}\right)-\left(\rho_{\varepsilon} * \nabla_{x^{\prime}} u_{1}\right)\left(x_{1}, x^{\prime}\right)\right) \mathrm{d} x^{\prime}\right| \\
& \leq c \varepsilon^{\frac{1}{N}}\left\|g_{1}\left(x_{1}, \cdot\right)\right\|_{L^{1}\left(\mathbb{R}^{N-1}\right)^{N}}\left\|D_{x^{\prime}}^{2} u_{1}\left(x_{1}, \cdot\right)\right\|_{L^{N}\left(\mathbb{R}^{N-1}\right)^{(N-1) \times(N-1)} .}
\end{aligned}
$$

Taking into account (A.2), (A.8), (A.9), we have thus proved that for any $x_{1} \in \mathbb{R}$,

$$
\begin{aligned}
\mid \int_{\mathbb{R}^{N-1}} f_{1}\left(x_{1}, x^{\prime}\right) & u_{1}\left(x_{1}, x^{\prime}\right) \mathrm{d} x^{\prime} \mid \\
& \leq c\left(\varepsilon^{\frac{1-N}{N}}\|g\|_{L^{1}\left(\mathbb{R}^{N}\right)^{N}}+\varepsilon^{\frac{1}{N}}\left\|g_{1}\left(x_{1}, \cdot\right)\right\|_{L^{1}\left(\mathbb{R}^{N-1}\right)^{N}}\right)\left\|D_{x^{\prime}}^{2} u_{1}\left(x_{1}, \cdot\right)\right\|_{L^{N}\left(\mathbb{R}^{N-1}\right)^{(N-1) \times(N-1)}} .
\end{aligned}
$$

If $\left\|g_{1}\left(x_{1}, \cdot\right)\right\|_{L^{1}\left(\mathbb{R}^{N-1}\right)^{N}} \neq 0$, we take

$$
\varepsilon:=\frac{\|g\|_{L^{1}\left(\mathbb{R}^{N}\right)^{N}}}{\left\|g_{1}\left(x_{1}, \cdot\right)\right\|_{L^{1}\left(\mathbb{R}^{N-1}\right)^{N}}} .
$$

Hence, we deduce from (A.10) that for any $x_{1} \in \mathbb{R}$,

$$
\left|\int_{\mathbb{R}^{N-1}} f_{1}\left(x_{1}, x^{\prime}\right) u_{1}\left(x_{1}, x^{\prime}\right) \mathrm{d} x^{\prime}\right| \leq c\|g\|_{L^{1}\left(\mathbb{R}^{N}\right)^{N \times N}}^{\frac{1}{N}}\left\|g_{1}\left(x_{1}, \cdot\right)\right\|_{L^{1}\left(\mathbb{R}^{N-1}\right)^{N}}^{\frac{1}{N^{\prime}}}\left\|D_{x^{\prime}}^{2} u_{1}\left(x_{1}, \cdot\right)\right\|_{L^{N}\left(\mathbb{R}^{N-1}\right)^{(N-1) \times(N-1)}} .
$$

Otherwise, we have $\left\|g_{1}\left(x_{1}, \cdot\right)\right\|_{L^{1}\left(\mathbb{R}^{N-1}\right)^{N}}=0$. Then, making $\varepsilon$ tend to infinity in (A.10) we obtain that (A.12) still holds true.

Therefore, integrating (A.12) with respect to $x_{1}$ and using Hölder's inequality in $\mathbb{R}$ with exponents $N^{\prime}, N$, it follows that

$$
\left|\int_{\mathbb{R}^{N}} f_{1} u_{1} \mathrm{~d} x\right| \leq C\|g\|_{L^{1}\left(\mathbb{R}^{N}\right)^{N \times N}}\left\|D^{2} u_{1}\right\|_{L^{N}\left(\mathbb{R}^{N}\right)^{N \times N}}
$$

which concludes the proof.

\section{A.2. Proof of Lemma 2.11}

In the sequel any point of $\mathbb{R}^{N}$ reads as $x=\left(x^{\prime}, x_{N}\right)$, with $x^{\prime} \in \mathbb{R}^{N-1}$ and $x_{N} \in \mathbb{R}$. The proof is divided into three steps:

First step: Extensions by reflection. Consider a tensor-valued $\mu \in C^{\infty}\left(\bar{B}(0, r)^{+}\right)^{N \times N}$ such that

$$
(\operatorname{Curl}(\operatorname{Div}(\mu)))_{i j}=\sum_{k, l=1}^{N} \partial_{k l}^{2} g_{i j k l}, \quad \text { in } \mathcal{D}^{\prime}\left(B(0, r)^{+}\right),
$$

where the fourth-order tensor-valued function $g$ is defined by

$$
g_{i j k l} \in C^{\infty}\left(\bar{B}(0, r)^{+}\right), \quad g_{i j k l}=-g_{j i k l}, \quad \text { for } i, j, k, l \in\{1, \ldots, N\} .
$$

We now extend $\mu$ and $g$ to $\bar{B}(0, r)$ thanks to reflections so that the transmission conditions hold through the boundary of $B(0, r)^{+}$. This needs to define the extended values to $B(0, r)^{-}$by a three-points interpolation (here, $\left.x_{N} / 3, x_{N} / 2, x_{N}\right)$ of the values in $B(0, r)^{+}$. A tedious computation we omit leads us to the following 
expressions of the extensions of $\mu$ and $g$ for $\left(x^{\prime}, x_{N}\right) \in B(0, r)^{-}$and $1 \leq i, j, k, l \leq N-1$,

$$
\begin{gathered}
\mu_{i j}\left(x^{\prime}, x_{N}\right):=3 \mu_{i j}\left(x^{\prime},-x_{N} / 3\right)-8 \mu_{i j}\left(x^{\prime},-x_{N} / 2\right)+6 \mu_{i j}\left(x^{\prime},-x_{N}\right), \\
\mu_{i N}\left(x^{\prime}, x_{N}\right):=-9 \mu_{i N}\left(x^{\prime},-x_{N} / 3\right)+16 \mu_{i N}\left(x^{\prime},-x_{N} / 2\right)-6 \mu_{i N}\left(x^{\prime},-x_{N}\right) \\
\mu_{N j}\left(x^{\prime}, x_{N}\right):=-\mu_{N j}\left(x^{\prime},-x_{N} / 3\right)+4 \mu_{N j}\left(x^{\prime},-x_{N} / 2\right)-6 \mu_{N j}\left(x^{\prime},-x_{N}\right) \\
\mu_{N N}\left(x^{\prime}, x_{N}\right):=3 \mu_{N N}\left(x^{\prime},-x_{N} / 3\right)-8 \mu_{N N}\left(x^{\prime},-x_{N} / 2\right)+6 \mu_{N N}\left(x^{\prime},-x_{N}\right) \\
g_{i j k l}\left(x^{\prime}, x_{N}\right):=3 g_{i j k l}\left(x^{\prime},-x_{N} / 3\right)-8 g_{i j k l}\left(x^{\prime},-x_{N} / 2\right)+6 g_{i j k l}\left(x^{\prime},-x_{N}\right) \\
g_{i j N l}\left(x^{\prime}, x_{N}\right):=-9 g_{i j N l}\left(x^{\prime},-x_{N} / 3\right)+16 g_{i j N l}\left(x^{\prime},-x_{N} / 2\right)-6 g_{i j N l}\left(x^{\prime},-x_{N}\right) \\
g_{i j k N}\left(x^{\prime}, x_{N}\right):=-9 g_{i j k N}\left(x^{\prime},-x_{N} / 3\right)+16 g_{i j k N}\left(x^{\prime},-x_{N} / 2\right)-6 g_{i j k N}\left(x^{\prime},-x_{N}\right) \\
g_{i j N N}\left(x^{\prime}, x_{N}\right):=27 g_{i j N l}\left(x^{\prime},-x_{N} / 3\right)-32 g_{i j N l}\left(x^{\prime},-x_{N} / 2\right)+6 g_{i j N l}\left(x^{\prime},-x_{N}\right) \\
g_{i N k l}\left(x^{\prime}, x_{N}\right):=-g_{i N k l}\left(x^{\prime},-x_{N} / 3\right)+4 g_{i N k l}\left(x^{\prime},-x_{N} / 2\right)-6 g_{i N k l}\left(x^{\prime},-x_{N}\right) \\
g_{i N N l}\left(x^{\prime}, x_{N}\right):=3 g_{i N N l}\left(x^{\prime},-x_{N} / 3\right)-8 g_{i N N l}\left(x^{\prime},-x_{N} / 2\right)+6 g_{i N N l}\left(x^{\prime},-x_{N}\right) \\
g_{i N k N}\left(x^{\prime}, x_{N}\right):=3 g_{i N k N}\left(x^{\prime},-x_{N} / 3\right)-8 g_{i N k N}\left(x^{\prime},-x_{N} / 2\right)+6 g_{i N k N}\left(x^{\prime},-x_{N}\right) \\
g_{i N N N}\left(x^{\prime}, x_{N}\right):=-9 g_{i N N N}\left(x^{\prime},-x_{N} / 3\right)+16 g_{i N N N}\left(x^{\prime},-x_{N} / 2\right)-6 g_{i N N N}\left(x^{\prime},-x_{N}\right) .
\end{gathered}
$$

Then, the matrix-valued measure $\mu$ and the functions $g_{i j k l}$ satisfy

$$
(\operatorname{Curl}(\operatorname{Div}(\mu)))_{i j}=\sum_{k, l=1}^{N} \partial_{k l}^{2} g_{i j k l} \quad \text { in } \mathcal{D}^{\prime}(B(0, r)) .
$$

Second step: A $W^{-1, N^{\prime}}$-estimate in the upper half ball. Let us prove that for any $s>1$ and any $r>0$, there exists a constant $C>0$ such that for any $\mu \in \mathcal{M}\left(B(0, r)^{+}\right)^{N \times N}$ satisfying $\operatorname{Curl}(\operatorname{Div}(\mu)) \in$ $W^{-2, s}\left(B(0, r)^{+}\right)$, we have

with

$$
\mu-\frac{1}{N} \operatorname{tr}(\mu) I_{N} \in W^{-1, N^{\prime}}\left(B(0, r / 2)^{+}\right)^{N \times N},
$$

$$
\left\|\mu-\frac{1}{N} \operatorname{tr}(\mu) I_{N}\right\|_{W^{-1, N^{\prime}}\left(B(0, r / 2)^{+}\right)} \leq C\left(\|\mu\|_{\mathcal{M}\left(B(0, r)^{+}\right)^{N \times N}}+\|\operatorname{Curl}(\operatorname{Div}(\mu))\|_{W^{-2, s}\left(B(0, r)^{+}\right)}\right) .
$$

To this end, given $\mu \in \mathcal{M}\left(B(0, r)^{+}\right)^{N \times N}$, with $\operatorname{Curl}(\operatorname{Div}(\mu)) \in W^{-2, s}\left(B(0, r)^{+}\right)^{N \times N}$, we consider functions $g_{i j k l} \in L^{s}\left(B(0, r)^{+}\right), 1 \leq i, j, k, l \leq N$, such that

$$
\begin{gathered}
g_{i j k l}=-g_{j i k l} \quad \text { in } \mathcal{D}^{\prime}\left(B(0, r)^{+}\right), \\
(\operatorname{Curl}(\operatorname{Div}(\mu)))_{i j}=\sum_{k, l=1}^{N} \partial_{k l}^{2} g_{i j k l} \quad \text { in } \mathcal{D}^{\prime}\left(B(0, r)^{+}\right), \\
\left(\sum_{k, l=1}^{N}\left\|g_{i j k l}\right\|_{L^{s}\left(B(0, r)^{+}\right)}^{s}\right)^{\frac{1}{s}}=\left\|(\operatorname{Curl}(\operatorname{Div}(\mu)))_{i j}\right\|_{W^{-2, s}\left(B(0, r)^{+}\right)} .
\end{gathered}
$$

For $0<h<r / 8$, define the regularizations $\mu^{h}$ and $g_{i j k l}^{h}$ in $C^{\infty}\left(\bar{B}(0,3 r / 4)^{+}\right)$of $\mu$ and $g_{i j k l}$, by

$$
\begin{gathered}
\mu^{h}(x):=\frac{1}{h^{N}} \int_{B\left(\left(x^{\prime}, x_{N}+h\right), h\right)} \rho\left(\frac{x^{\prime}-y^{\prime}}{h}, \frac{x_{N}-y_{N}+h}{h}\right) \mathrm{d} \mu(y), \quad \text { for } x \in \bar{B}(0,3 r / 4)^{+}, \\
g_{i j k l}^{h}(x)=\frac{1}{h^{N}} \int_{B\left(\left(x^{\prime}, x_{N}+h\right), h\right)} \rho\left(\frac{x^{\prime}-y^{\prime}}{h}, \frac{x_{N}-y_{N}+h}{h}\right) g_{i j k l}(y) \mathrm{d} y, \quad \text { for } x \in \bar{B}(0,3 r / 4)^{+},
\end{gathered}
$$


where $\rho$ denotes a mollifier in $C_{c}^{\infty}(B(0,1))$, with integral 1 . Then, $\mu^{h}$ and the functions $g_{i j k l}^{h}$ are related by the equality

$$
\left(\operatorname{Curl}\left(\operatorname{Div}\left(\mu^{h}\right)\right)\right)_{i j}=\sum_{k, l=1}^{N} \partial_{k l}^{2} g_{i j k l}^{h} \quad \text { in } \mathcal{D}^{\prime}\left(B(0,3 r / 4)^{+}\right) .
$$

Using the first step with $r$ replaced by $3 r / 4$, we can construct extensions of $\mu^{h}$ and $g_{i j k l}^{h}$ to $B(0,3 r / 4)$, still denoted by $\mu^{h}$ and $g_{i j k l}^{h}$, such that

$$
\begin{gathered}
\left\|\mu^{h}\right\|_{\mathcal{M}(B(0,3 r / 4))} \leq C\left\|\mu^{h}\right\|_{\mathcal{M}\left(B(0,3 r / 4)^{+}\right)} \leq C\|\mu\|_{\mathcal{M}\left(B(0, r)^{+}\right)}, \\
\left\|g_{i j k l}^{h}\right\|_{L^{s}(B(0,3 r / 4))} \leq C\left\|g_{i j k l}^{h}\right\|_{L^{s}\left(B(0,3 r / 4)^{+}\right)} \leq C\left\|g_{i j k l}\right\|_{L^{s}\left(B(0, r)^{+}\right)}, \\
\left(\operatorname{Curl}\left(\operatorname{Div}\left(\mu^{h}\right)\right)\right)_{i j}=\sum_{k, l=1}^{N} \partial_{k l}^{2} g_{i j k l}^{h} \quad \text { in } \mathcal{D}^{\prime}(B(0,3 r / 4)) .
\end{gathered}
$$

Using the linearity and the continuity of the regularization and of the extension operators, we obtain extensions of $\mu$ and $g_{i j k l}$ to $B(0,3 r / 4)$, still denoted by $\mu$ and $g_{i j k l}$, such that

$$
\begin{gathered}
\|\mu\|_{\mathcal{M}(B(0,3 r / 4))} \leq C\|\mu\|_{\mathcal{M}\left(B(0, r)^{+}\right)}, \quad\left\|g_{i j k l}\right\|_{L^{s}(B(0,3 r / 4))} \leq C\left\|g_{i j k l}\right\|_{L^{s}\left(B(0, r)^{+}\right)}, \\
(\operatorname{Curl}(\operatorname{Div}(\mu)))_{i j}=\sum_{k, l=1}^{N} \partial_{k l}^{2} g_{i j k l} \quad \text { in } \mathcal{D}^{\prime}(B(0,3 r / 4)) .
\end{gathered}
$$

Now, consider $\varphi \in C_{c}^{\infty}(B(0,3 r / 4))$ such that $\varphi=1$ in $B(0, r / 2)$. Given $t \in\left(1, N^{\prime}\right)$ with $t \leq s$, the matrixvalued function $\varphi \mu$ satisfies $\operatorname{Curl}(\operatorname{Div}(\varphi \mu)) \in W^{-2, t}(B(0,3 r / 4))^{N \times N}$, with

$$
\|\operatorname{Curl}(\operatorname{Div}(\varphi \mu))\|_{W^{-2, t}(B(0,3 r / 4))^{N \times N}} \leq C\left(\|\mu\|_{\mathcal{M}\left(B(0, r)^{+}\right)^{N \times N}}+\|\operatorname{Curl}(\operatorname{Div}(\mu))\|_{W^{-2, s}\left(B(0, r)^{+}\right)^{N \times N}}\right) .
$$

On the other hand, consider the solutions $u$ and $p$ of the Stokes problem

$$
\begin{cases}-\Delta u+\nabla p=-\operatorname{Div}(\varphi \mu) & \text { in } \mathbb{R}^{N} \\ \operatorname{div}(u)=0 & \text { in } \mathbb{R}^{N}\end{cases}
$$

which are given by

$$
p=-\sum_{j, k=1}^{N} \partial_{j k}^{2} \Gamma *\left(\varphi \mu_{j k}\right), \quad u_{i}=\sum_{j=1}^{N} \partial_{j}(\Gamma * \Gamma) *(\operatorname{Curl}(\operatorname{Div}(\varphi \mu)))_{i j}, \quad \text { for } i \in\{1, \ldots, N\} .
$$

Then, proceeding as in the proof of Theorem 2.7 for deriving (2.49) thanks to the Calderón-Zygmund estimates (1.6) and (1.8), and using (A.15) it follows that $D u$ is in $L^{t}(B(0,3 r / 4))^{N \times N}$, with

$$
\begin{aligned}
\|D u\|_{L^{t}(B(0,3 r / 4))^{N \times N}} & \leq C\|\operatorname{Curl}(\operatorname{Div}(\varphi \mu))\|_{W^{-2, t}(B(0,3 r / 4))^{N \times N}} \\
& \leq C\left(\|\mu\|_{\mathcal{M}\left(B(0, r)^{+}\right)^{N \times N}}+\|\operatorname{Curl}(\operatorname{Div}(\mu))\|_{W^{-2, s}\left(B(0, r)^{+}\right)^{N \times N}}\right) .
\end{aligned}
$$

Moreover, by the Stokes equation $\varphi \mu-D u$ clearly satisfies

$$
\operatorname{Curl}(\operatorname{Div}(\varphi \mu-D u))=0 \quad \text { in } \mathcal{D}^{\prime}(B(0,3 r / 4))^{N \times N} .
$$

Therefore, applying Theorem 2.7 with the open sets $\Omega:=B(0,3 r / 4)$ and $\omega:=B(0, r / 2)$, and using that $\varphi=1$ in $B(0, r / 2), \operatorname{tr}(D u)=\operatorname{div}(u)=0$ and $(\mathrm{A} .16)$, we get that $\mu-D u-\frac{1}{N} \operatorname{tr}(\mu) I_{N}$ belongs to $W^{-1, N^{\prime}}(B(0, r / 2))^{N \times N}$, 
with

$$
\begin{aligned}
& \left\|\mu-D u-\frac{1}{N} \operatorname{tr}(\mu) I_{N}\right\|_{W^{-1, N^{\prime}}(B(0, r / 2))^{N \times N}} \leq C\|\varphi \mu-D u\|_{\mathcal{M}(B(0,3 r / 4))^{N \times N}} \\
& \leq C\left(\|\mu\|_{\mathcal{M}\left(B(0, r)^{+}\right)^{N \times N}}+\|\operatorname{Curl}(\operatorname{Div}(\mu))\|_{W^{-2, s}\left(B(0, r)^{+}\right)^{N \times N}}\right) .
\end{aligned}
$$

Finally, using the embedding of $L^{t}(B(0, r))$ into $W^{-1, N^{\prime}}(B(0, r))\left(t<N^{\prime}\right)$, we deduce estimate (A.14) from the last inequality again with (A.16).

Third step: Proof of Lemma 2.11. Let $\mu$ be in $\mathcal{M}\left(\Theta^{+}\right)^{N}$, with $\operatorname{Curl}(\operatorname{Div}(\mu))=0$ in $\mathcal{D}^{\prime}\left(\Theta^{+}\right)^{N \times N}$. Denoting by $\mu_{i}, 1 \leq i \leq N$, the rows of $\mu$, consider the matrix-valued measure $\hat{\mu} \in \mathcal{M}\left(B(0, r)^{+}\right)$with rows $\hat{\mu}_{i}$, defined by

$$
\int_{B(0, r)^{+}} \hat{\Phi}(y) \cdot \mathrm{d} \hat{\mu}_{i}(y)=\sum_{j=1}^{N} \int_{\Theta^{+}} \hat{\Phi}\left(\psi^{-1}(x)\right) \cdot \partial_{y_{i}} \psi_{j}\left(\psi^{-1}(x)\right)\left(D\left(\psi^{-1}\right)(x)\right)^{T} \operatorname{det}\left(D\left(\psi^{-1}\right)(x)\right) \mathrm{d} \mu_{j}(x)
$$

for any $\hat{\Phi} \in C_{0}^{0}\left(B(0, r)^{+}\right)^{N}$. Note that if $\mu \in L^{1}\left(\Theta^{+}\right)$, we simply have

$$
\hat{\mu}_{i}(y)=\sum_{j=1}^{N} \partial_{y_{i}} \psi_{j}(y)\left((D \psi)^{-1}(y)\right)^{T} \mu_{j}(\psi(y)), \quad \text { a.e. } y \in B(0, r)^{+}
$$

Thanks to the $C^{3}$-regularity of $\psi$ and to the assumption on $\mu$, an easy computation leads to

$$
\operatorname{Curl}(\operatorname{Div}(\hat{\mu}))=A(y) \hat{\mu}+B(y) D \hat{\mu}
$$

where $A \in C^{0}\left(\bar{B}(0, r)^{+}\right)^{N^{2} \times N^{2}}$ and $B \in C^{1}\left(\bar{B}(0, r)^{+}\right)^{N^{2} \times N^{3}}$. Therefore, for any $s \in\left(1, N^{\prime}\right)$, Curl $(\operatorname{Div}(\hat{\mu}))$ belongs to $W^{-2, s}\left(B(0, r)^{+}\right)$, with

$$
\|\operatorname{Curl}(\operatorname{Div}(\hat{\mu}))\|_{W^{-2, s}\left(B(0, r)^{+}\right)^{N \times N}} \leq C\|\hat{\mu}\|_{\mathcal{M}\left(B(0, r)^{+}\right)^{N \times N}} .
$$

Then, from the second step we deduce that $\hat{\mu}-\frac{1}{N} \operatorname{tr}(\hat{\mu}) I_{N}$ belongs to $W^{-1, N^{\prime}}\left(B(0, r / 2)^{+}\right)$, with

$$
\left\|\hat{\mu}-\frac{1}{N} \operatorname{tr}(\hat{\mu}) I_{N}\right\|_{W^{-1, N^{\prime}}\left(B(0, r / 2)^{+}\right)^{N \times N}} \leq C\|\hat{\mu}\|_{\mathcal{M}\left(B(0, r)^{+}\right)^{N \times N}} .
$$

Finally, similarly to (A.17) we have

$$
\begin{aligned}
\int_{\tilde{\Theta}^{+}} \Phi(x) \cdot d\left(\mu_{i}-\frac{1}{N} \mu_{i i} e_{i}\right) & (x) \\
= & \sum_{j=1}^{N} \int_{B(0, r / 2)^{+}} \Phi(\psi(y)) \cdot \partial_{x_{i}}\left(\psi^{-1}\right)_{j}(\psi(y))(D \psi(y))^{T} \operatorname{det}(D \psi(y)) d\left(\hat{\mu}_{j}-\frac{1}{N} \hat{\mu}_{j j} e_{j}\right)(y)
\end{aligned}
$$

for any $\Phi \in C_{0}^{0}\left(\tilde{\Theta}^{+}\right)^{N}$. This combined with $\left(\right.$ A.18) implies that $\mu-\frac{1}{N} \operatorname{tr}(\mu) I_{N}$ belongs to $W^{-1, N^{\prime}}\left(\tilde{\Theta}^{+}\right)^{N \times N}$ and that (2.54) holds.

Acknowledgements. The authors wish to thank the unknown referees for several suggestions and comments which led to improvements. The authors have been partially supported by the project MTM2008-00306 of the "Ministerio de Ciencia e Innovación" of Spain. Juan Casado Díaz is grateful for support from the "Institut National des Sciences Appliquées de Rennes" during his visit in July 2009. Marc Briane thanks the University of Sevilla for its hospitality in September 2009. 


\section{REFERENCES}

[1] C. Amrouche and V. Girault, Decomposition of vector spaces and application to the Stokes problem in arbitrary dimension. Czechoslovak Math. J. 44 (1994) 109-140.

[2] M. Bellieud and G. Bouchitté, Homogenization of elliptic problems in a fiber reinforced structure. Nonlocal effects. Ann. Scuola Norm. Sup. Pisa Cl. Sci. 26 (1998) 407-436.

[3] M.E. Bogovski, Solution of the first boundary value problem for the equation of continuity of an incompressible medium. Soviet Math. Dokl. 20 (1979) 1094-1098.

[4] J. Bourgain and H. Brezis, New estimates for elliptic equations and Hodge type systems. J. Eur. Math. Soc. 9 (2007) $277-315$.

[5] H. Brezis, Analyse Fonctionnelle, Théorie et Applications. Mathématiques Appliquées pour la Maîtrise, Masson, Paris (1983).

[6] H. Brezis and J. Van Schaftingen, Boundary estimates for elliptic systems with $L^{1}$-data. Calc. Var. 30 (2007) 369-388.

[7] M. Briane, Homogenization of the Stokes equations with high-contrast viscosity. J. Math. Pures Appl. 82 (2003) 843-876.

[8] M. Briane and J. Casado Díaz, Compactness of sequences of two-dimensional energies with a zero-order term. Application to three-dimensional nonlocal effects. Calc. Var. 33 (2008) 463-492.

[9] M. Camar-Eddine and P. Seppecher, Determination of the closure of the set of elasticity functionals. Arch. Rat. Mech. Anal. 170 (2003) 211-245.

[10] G. de Rham, Variétés différentiables, Formes, courants, formes harmoniques. Hermann, Paris (1973).

[11] D. Gilbarg and N. Trudinger, Elliptic Partial Differential Equations of Second Order. Springer, Berlin (2001).

[12] E. Hopf, Über die Anfwangswertaufgabe für die hydrodynamischen Grundgleichungen. Math. Nachr. 4 (1951) 213-231.

[13] E.Y. Khruslov, Homogenized models of composite media, in Composite Media and Homogenization Theory, G. Dal Maso and G.F. Dell'Antonio Eds., Progress in Nonlinear Differential Equations and Their Applications, Birkhäuser (1991) 159-182.

[14] O.A. Ladyzhenskaya, The mathematical theory of viscous incompressible flow, Mathematics and its Applications 2 . Gordon and Breach, Science Publishers, New York-London-Paris (1969).

[15] J.-L. Lions, Quelques résultats d'existence dans des équations aux dérivées partielles non linéaires. Bull. S.M.F. 87 (1959) $245-273$.

[16] V.A. Marchenko and E.Y. Khruslov, Homogenization of partial differential equations, Progress in Mathematical Physics 46. Birkhäuser, Boston (2006).

[17] J. Nečas, Équations aux dérivées partielles. Presses de l'Université de Montréal (1965).

[18] C. Pideri and P. Seppecher, A second gradient material resulting from the homogenization of an heterogeneous linear elastic medium. Continuum Mech. Thermodyn. 9 (1997) 241-257.

[19] M.-J. Strauss, Variations of Korn's and Sobolev's inequalities, in Partial Differential Equations: Proc. Symp. Pure Math. 23, D. Spencer Ed., Am. Math. Soc., Providence (1973) 207-214.

[20] L. Tartar, Topics in nonlinear analysis. Publications Mathématiques d'Orsay 78 (1978) 271.

[21] R. Temam, Navier-Stokes Equations - Theory and Numerical Analysis, Studies in Mathematics and its Applications 2. North-Holland, Amsterdam (1984).

[22] J. Van Schaftingen, Estimates for $L^{1}$-vector fields under higher-order differential conditions. J. Eur. Math. Soc. 10 (2008) 867-882.

[23] J. Van Schaftingen, Estimates for $L^{1}$-vector fields. C. R. Acad. Sci. Paris, Ser. I 339 (2004) 181-186. 